TRANSACTIONS OF THE

AMERICAN MATHEMATICAL SOCIETY

Volume 362, Number 12, December 2010, Pages 6425-6455

S 0002-9947(2010)05290-0

Article electronically published on July 23, 2010

\title{
ON ALMOST UNIVERSAL MIXED SUMS OF SQUARES AND TRIANGULAR NUMBERS
}

\author{
BEN KANE AND ZHI-WEI SUN
}

\begin{abstract}
In 1997 K. Ono and K. Soundararajan [Invent. Math. 130(1997)] proved that under the generalized Riemann hypothesis any positive odd integer greater than 2719 can be represented by the famous Ramanujan form $x^{2}+$ $y^{2}+10 z^{2}$; equivalently the form $2 x^{2}+5 y^{2}+4 T_{z}$ represents all integers greater than 1359 , where $T_{z}$ denotes the triangular number $z(z+1) / 2$. Given positive integers $a, b, c$ we employ modular forms and the theory of quadratic forms to determine completely when the general form $a x^{2}+b y^{2}+c T_{z}$ represents sufficiently large integers and to establish similar results for the forms $a x^{2}+$ $b T_{y}+c T_{z}$ and $a T_{x}+b T_{y}+c T_{z}$. Here are some consequences of our main theorems: (i) All sufficiently large odd numbers have the form $2 a x^{2}+y^{2}+z^{2}$ if and only if all prime divisors of $a$ are congruent to 1 modulo 4. (ii) The form $a x^{2}+y^{2}+T_{z}$ is almost universal (i.e., it represents sufficiently large integers) if and only if each odd prime divisor of $a$ is congruent to 1 or 3 modulo 8. (iii) $a x^{2}+T_{y}+T_{z}$ is almost universal if and only if all odd prime divisors of $a$ are congruent to 1 modulo 4 . (iv) When $v_{2}(a) \neq 3$, the form $a T_{x}+T_{y}+T_{z}$ is almost universal if and only if all odd prime divisors of $a$ are congruent to 1 modulo 4 and $v_{2}(a) \neq 5,7, \ldots$, where $v_{2}(a)$ is the 2 -adic order of $a$.
\end{abstract}

\section{INTRODUCTION AND THE MAIN RESUlTS}

A classical theorem of Lagrange states that any $n \in \mathbb{N}=\{0,1,2, \ldots\}$ can be written as a sum of four squares (of integers). In 1916 S. Ramanujan 22 found all the finitely many vectors $(a, b, c, d)$ with $a, b, c, d \in \mathbb{Z}^{+}=\{1,2,3, \ldots\}$ such that the form $a x^{2}+b y^{2}+c z^{2}+d w^{2}$ (with $x, y, z, w \in \mathbb{Z}$ ) represents all natural numbers. Ramanujan also asked for determining those vectors $(a, b, c, d) \in\left(\mathbb{Z}^{+}\right)^{4}$ such that the form $a x^{2}+b y^{2}+c z^{2}+d w^{2}$ represents all sufficiently large integers; this problem was essentially solved by H. D. Kloosterman [13] with help from the useful Kloosterman sum, and this work represents a major breakthrough in the field of quadratic forms.

What about ternary quadratic forms? A well-known theorem of Gauss and Legendre states that $n \in \mathbb{N}$ is a sum of three squares if and only if it is not of the form $4^{k}(8 l+7)$ with $k, l \in \mathbb{N}$. In general, it is known that for any $a, b, c \in \mathbb{Z}^{+}$the

Received by the editors September 18, 2008.

2010 Mathematics Subject Classification. Primary 11E25; Secondary 11D85, 11E20, 11E95, 11F27, 11F37, 11P99, 11S99.

Key words and phrases. Representations of integers, triangular numbers, sums of squares, quadratic forms, half-integral weight modular forms.

This research was conducted when the first author was a postdoctor at Radboud Universiteit, Nijmegen, Netherlands.

The second author was the corresponding author and he was supported by the National Natural Science Foundation (grant 10871087) of the People's Republic of China.

(C)2010 American Mathematical Society 6425

Reverts to public domain 28 years from publication 
subset $\left\{a x^{2}+b y^{2}+c z^{2}: x, y, z \in \mathbb{Z}\right\}$ of $\mathbb{N}$ cannot have asymptotic density 1 because there is always a congruence class modulo a power of some prime $p$ dividing $2 a b c$ which is not even locally represented by the form $a x^{2}+b y^{2}+c z^{2}$.

For $x \in \mathbb{Z}$ let $T_{x}$ denote the triangular number $x(x+1) / 2$. Clearly $T_{n}=T_{-n-1}$ for all $n \in \mathbb{N}$. A famous assertion of Fermat states that each $n \in \mathbb{N}$ can be expressed as a sum of three triangular numbers, equivalently $8 n+3$ is a sum of three (odd) squares; this follows immediately from the Gauss-Legendre theorem. Here is another consequence of the Gauss-Legendre theorem observed by Euler: Each natural number can be written in the form $x^{2}+y^{2}+T_{z}$ with $x, y, z \in \mathbb{Z}$. Recently, B. K. Oh and the second author [16] showed that for any $n \in \mathbb{Z}^{+}$there are $x, y, z \in \mathbb{Z}$ such that $n=x^{2}+(2 y+1)^{2}+T_{z}$, i.e., $n-1=x^{2}+8 T_{y}+T_{z}$.

In view of the above, it is natural to study mixed sums of squares and triangular numbers of the following three types:

$$
a x^{2}+b y^{2}+c T_{z}, a x^{2}+b T_{y}+c T_{z}, a T_{x}+b T_{y}+c T_{z},
$$

where $a, b, c \in \mathbb{Z}^{+}=\{1,2,3, \ldots\}$. Let $f(x, y, z)$ be any of the three forms, and define the exceptional set

$$
E(f):=\{n \in \mathbb{N}: f(x, y, z)=n \text { has no integral solution }\} .
$$

If $E(f)=\emptyset$, then $f$ is said to be universal; if $E(f)$ is finite, then we call $f$ almost universal. When the set $E(f)$ has asymptotic density zero, i.e.,

$$
\lim _{N \rightarrow+\infty} \frac{\mid\{1 \leq n \leq N: f(x, y, z)=n \text { for some } x, y, z \in \mathbb{Z}\} \mid}{N}=1,
$$

we say that $f$ is asymptotically universal. In the case $\operatorname{gcd}(a, b, c)>1$, obviously $f$ is neither almost universal nor asymptotically universal.

In 1862 J. Liouville (cf. 4, p. 23]) proved the following result: For positive integers $a \leq b \leq c$, the form $a T_{x}+b T_{y}+c T_{z}$ is universal if and only if $(a, b, c)$ is among the following vectors:

$$
(1,1,1),(1,1,2), \quad(1,1,4),(1,1,5),(1,2,2),(1,2,3),(1,2,4) .
$$

Recently the second author 27 initiated the determination of all universal forms of the type $a x^{2}+b y^{2}+c T_{z}$ or $a x^{2}+b T_{y}+c T_{z}$, and the project was finally completed by combining the results in [27, 9] and [16. Namely, for $a, b, c \in \mathbb{Z}^{+}$with $a \leq b$, the form $a x^{2}+b y^{2}+c T_{z}$ is universal if and only if $(a, b, c)$ is among the following vectors:

$$
\begin{aligned}
& (1,1,1),(1,1,2),(1,2,1),(1,2,2),(1,2,4), \\
& (1,3,1),(1,4,1),(1,4,2),(1,8,1),(2,2,1) .
\end{aligned}
$$

Also, for $a, b, c \in \mathbb{Z}^{+}$with $b \geq c$, the form $a x^{2}+b T_{y}+c T_{z}$ is universal if and only if $(a, b, c)$ is among the following vectors:

$$
\begin{aligned}
& (1,1,1),(1,2,1),(1,2,2),(1,3,1),(1,4,1),(1,4,2),(1,5,2), \\
& (1,6,1),(1,8,1),(2,1,1),(2,2,1),(2,4,1),(3,2,1),(4,1,1),(4,2,1) .
\end{aligned}
$$

In 1916 Ramanujan (cf. 22] and 19]) conjectured that those positive even integers not represented by $x^{2}+y^{2}+10 z^{2}$ are exactly those of the form $4^{k}(16 l+6)$ with $k, l \in \mathbb{N}$ and that those positive odd integers not represented by $x^{2}+y^{2}+10 z^{2}$ are as follows:

$$
3,7,21,31,33,43,67,79,87,133,217,219,223,253,307,391, \ldots .
$$


In 1927 L. E. Dickson [3] proved Ramanujan's conjecture about even numbers by a simple argument. However, Ramanujan's conjecture about odd numbers is very difficult. For $n \in \mathbb{N}$, clearly

$$
\begin{aligned}
& 2 n+1=x^{2}+y^{2}+10 z^{2} \text { for some } x, y, z \in \mathbb{Z} \\
\Longleftrightarrow & 2 n+1=(2 x)^{2}+10 y^{2}+(2 z+1)^{2} \text { for some } x, y, z \in \mathbb{Z} \\
\Longleftrightarrow & n=2 x^{2}+5 y^{2}+4 T_{z} \text { for some } x, y, z \in \mathbb{Z} .
\end{aligned}
$$

Only in 1990 were W. Duke and R. Schulze-Pillot $[6$ able to show that sufficiently large odd integers can be written in the form $x^{2}+y^{2}+10 z^{2}$, or equivalently that the form $2 x^{2}+5 y^{2}+4 T_{z}$ is almost universal. In $1997 \mathrm{~K}$. Ono and K. Soundararajan 20 showed further that the generalized Riemann hypothesis implies that the only positive odd integers not in the form $x^{2}+y^{2}+10 z^{2}$ are those listed by Ramanujan together with 679 and 2719 ; in other words $E\left(2 x^{2}+5 y^{2}+4 T_{z}\right)$ consists of the following numbers:

1, 3, 10, 15, 16, 21, 33, 39, 43, 66, 108, 109, 111, 126, 153, 195, 339, 1359.

Motivated by his conjecture on sums of primes and triangular numbers (cf. 29, Conjecture 1.1]), the second author [28] conjectured that for any $k, l \in \mathbb{N}$ the form $2^{k} x^{2}+2^{l} y^{2}+T_{z}$ is almost universal. The first author 12 showed that all of those forms conjectured to be almost universal in 28, are asymptotically universal and that many of them are almost universal.

In this paper we aim at determining all asymptotically universal forms and almost universal forms of the three types via modular forms and the theory of quadratic forms.

For convenience we introduce some basic notation. We may write a positive integer $a$ in the form $2^{v_{2}(a)} a^{\prime}$ with $v_{2}(a) \in \mathbb{N}$ and $a^{\prime}$ odd; $v_{2}(a)$ is called the 2-adic order of $a$ (equivalently, $2^{v_{2}(a)} \| a$ ), while $a^{\prime}$ is said to be the odd part of $a$. For $a \in \mathbb{Z}$ and $m \in \mathbb{Z}^{+}$, by $a R m$ we mean that $a$ is quadratic residue modulo $m$, i.e., $a$ is relatively prime to $m$ and the equation $x^{2} \equiv a(\bmod m)$ is solvable over $\mathbb{Z}$. For an integer $a$ and a positive odd integer $m$, it is well known that $a R m$ if and only if the Legendre symbol $\left(\frac{a}{p}\right)$ equals 1 for every prime divisor $p$ of $m$.

Now we state our results on asymptotically universal forms.

Theorem 1.1. Fix $a, b, c \in \mathbb{Z}^{+}$with $\operatorname{gcd}(a, b, c)=1$. Then the form

$$
f(x, y, z):=a x^{2}+b y^{2}+c T_{z}
$$

is asymptotically universal if and only if we have the following:

(1) $-2 b c R a^{\prime},-2 a c R b^{\prime}$, and - ab $R c^{\prime}$.

(2) Either $4 \nmid c$, or both $4 \| c$ and $2 \| a b$.

Theorem 1.2. Fix $a, b, c \in \mathbb{Z}^{+}$with $\operatorname{gcd}(a, b, c)=1$. Then the form

$$
f(x, y, z):=a x^{2}+b T_{y}+c T_{z}
$$

is asymptotically universal if and only if we have the following:

(1) $-b c R a^{\prime},-2 a c R b^{\prime}$, and - 2abR $c^{\prime}$.

(2) Either $4 \nmid b$ or $4 \nmid c$.

Theorem 1.3. Fix $a, b, c \in \mathbb{Z}^{+}$with $\operatorname{gcd}(a, b, c)=1$. Then the form

$$
f(x, y, z):=a T_{x}+b T_{y}+c T_{z}
$$


is asymptotically universal if and only if

$$
-b c R a^{\prime},-a c R b^{\prime} \text {, and }-a b R c^{\prime} \text {. }
$$

Remark 1.1. For $a, b, c \in \mathbb{Z}^{+}$, if the form $a x^{2}+b y^{2}+c T_{z}$ or $a x^{2}+b T_{y}+c T_{z}$ or $a T_{x}+b T_{y}+c T_{z}$ is asymptotically universal, then $a^{\prime}, b^{\prime}, c^{\prime}$ must be pairwise coprime by Theorems 1.1-1.3.

The law of quadratic reciprocity gives restrictions under which the relations in the above theorems cannot occur.

Corollary 1.4. Fix $a, b, c \in \mathbb{Z}^{+}$and consider

$$
\begin{array}{lll}
\text { (1) } a x^{2}+b y^{2}+c T_{z}, & \text { (2) } a T_{x}+b T_{y}+c z^{2}, & \text { (3) } a T_{x}+b T_{y}+c T_{z}, \\
& \text { (4) } a x^{2}+b T_{y}+c z^{2}, & \text { (5) } a x^{2}+b T_{y}+c T_{z} .
\end{array}
$$

(i) If $a^{\prime} \equiv b^{\prime} \equiv-c^{\prime}(\bmod 8)$, then none of (1)-(5) is asymptotically universal.

(ii) If

$$
\left\{\begin{array} { l } 
{ a ^ { \prime } \equiv b ^ { \prime } \equiv c ^ { \prime } + 4 \quad ( \operatorname { m o d } 8 ) , } \\
{ v _ { 2 } ( a ) \not \equiv v _ { 2 } ( b ) \quad ( \operatorname { m o d } 2 ) }
\end{array} \quad \text { or } \quad \left\{\begin{array}{l} 
\pm a^{\prime} \equiv-b^{\prime} \equiv c^{\prime}+4 \quad(\bmod 8) \\
v_{2}(a) \equiv v_{2}(b) \quad(\bmod 2)
\end{array}\right.\right.
$$

then none of (1)-(3) is asymptotically universal.

(iii) If

$$
\left\{\begin{array} { l } 
{ a ^ { \prime } \equiv b ^ { \prime } \equiv c ^ { \prime } + 4 \quad ( \operatorname { m o d } 8 ) , } \\
{ v _ { 2 } ( a ) \equiv v _ { 2 } ( b ) \quad ( \operatorname { m o d } 2 ) }
\end{array} \quad \text { or } \quad \left\{\begin{array}{l} 
\pm a^{\prime} \equiv-b^{\prime} \equiv c^{\prime}+4 \quad(\bmod 8) \\
v_{2}(a) \not \equiv v_{2}(b) \quad(\bmod 2)
\end{array}\right.\right.
$$

then neither (4) nor (5) is asymptotically universal.

Corollary 1.5. Let $a, b, c \in \mathbb{Z}^{+}$with $v_{2}(b) \equiv v_{2}(c)(\bmod 2)$. Assume that $a^{\prime} \equiv b^{\prime} \equiv$ $c^{\prime}(\bmod 4)$ fails. Then, either none of the forms $a x^{2}+b y^{2}+2 c T_{z}$ and $a x^{2}+c y^{2}+2 b T_{z}$ is asymptotically universal or none of the forms $a x^{2}+2 c y^{2}+b T_{z}$ and $a x^{2}+2 b y^{2}+c T_{z}$ is asymptotically universal.

Any $n \in \mathbb{Z}^{+}$can be uniquely written in the form $a^{2} q$ with $a, q \in \mathbb{Z}^{+}$and $q$ squarefree, and we use $\mathcal{S F}(n)$ to denote $q=\prod_{p \mid n, 2 \nmid v_{p}(n)} p$, the squarefree part of $n$.

Now we turn to almost universal forms.

Theorem 1.6. Let $a, b, c \in \mathbb{Z}^{+}$with $\operatorname{gcd}(a, b, c)=1$ and $v_{2}(a) \geq v_{2}(b)$. Suppose that both (1) and (2) in Theorem 1.1 hold. Then there are infinitely many positive integers not represented by the form

$$
f(x, y, z):=a x^{2}+b y^{2}+c T_{z}
$$

(i.e., $f$ is not almost universal) if and only if we have the following:

(1) $2 \mid a, 4 \nmid c, a^{\prime} \equiv b^{\prime}\left(\bmod 2^{3-v_{2}(c)}\right)$, and

$$
\left\{\begin{array}{l}
4 \nmid b \Rightarrow v_{2}(a) \equiv c(\bmod 2), \\
2 \nmid b c \Rightarrow 8|a \& 8|(b-c) .
\end{array}\right.
$$

(2) All prime divisors of $\mathcal{S} \mathcal{F}\left(a^{\prime} b^{\prime} c^{\prime}\right)$ are congruent to 1 modulo 4 if $v_{2}(a) \equiv$ $v_{2}(b)(\bmod 2)$, and congruent to 1 or 3 modulo 8 otherwise.

(3) $2^{3-v_{2}(c)}\left(a x^{2}+b y^{2}\right)+c^{\prime} z^{2}=\mathcal{S F}\left(a^{\prime} b^{\prime} c^{\prime}\right)$ has no integral solutions. 
Remark 1.2. When $a x^{2}+b y^{2}+c T_{z}$ (with $a, b, c \in \mathbb{Z}^{+}$) is asymptotically universal it is not necessary that (2) in Theorem 1.6 holds. For example, $6 x^{2}+y^{2}+10 T_{z}$ is asymptotically universal, but we don't have (2) in Theorem 1.6 with $a=6, b=1$ and $c=10$.

Example 1.1. Consider those forms $a x^{2}+b y^{2}+c T_{z}$ with $a, b, c \in \mathbb{Z}^{+}$and $a+b+c \leq$ 10. By Theorem 1.6] we find that those asymptotically universal ones are all almost universal. Below is a complete list of those forms $a x^{2}+b y^{2}+c T_{z}$ with $a, b, c \in \mathbb{Z}^{+}$ and $a+b+c \leq 10$ which are almost universal but not universal:

$$
\begin{aligned}
& x^{2}+2 y^{2}+3 T_{z}, 2 x^{2}+4 y^{2}+T_{z}, x^{2}+5 y^{2}+2 T_{z}, x^{2}+6 y^{2}+T_{z}, \\
& x^{2}+y^{2}+5 T_{z}, 2 x^{2}+3 y^{2}+2 T_{z}, 2 x^{2}+5 y^{2}+T_{z}, 3 x^{2}+4 y^{2}+T_{z}, \\
& x^{2}+2 y^{2}+6 T_{z}, x^{2}+5 y^{2}+3 T_{z}, 2 x^{2}+2 y^{2}+5 T_{z}, 2 x^{2}+4 y^{2}+3 T_{z}, \\
& 4 x^{2}+4 y^{2}+T_{z}, x^{2}+4 y^{2}+5 T_{z}, 2 x^{2}+3 y^{2}+5 T_{z} .
\end{aligned}
$$

For the four forms in the first row, the second author [27] conjectured that

$$
\begin{aligned}
& E\left(x^{2}+2 y^{2}+3 T_{z}\right)=\{23\}, E\left(2 x^{2}+4 y^{2}+T_{z}\right)=\{20\}, \\
& E\left(x^{2}+5 y^{2}+2 T_{z}\right)=\{19\}, E\left(x^{2}+6 y^{2}+T_{z}\right)=\{47\},
\end{aligned}
$$

which was confirmed by the first author [12] under the generalized Riemann hypothesis. For the form $4 x^{2}+4 y^{2}+T_{z}$ the second author [29] conjectured that $E\left(4 x^{2}+4 y^{2}+T_{z}\right)$ consists of the following 19 numbers:

$2,12,13,24,27,34,54,84,112,133$,

162, 234, 237, 279, 342, 399, 652, 834, 864 .

For the ten remaining forms on the above list, our computation via computer suggests the following information:

$$
\begin{aligned}
& E\left(x^{2}+y^{2}+5 T_{z}\right)=\{3,11,12,27,129,138,273\}, \\
& E\left(2 x^{2}+3 y^{2}+2 T_{z}\right)=\{1,19,43,94\}, E\left(2 x^{2}+5 y^{2}+T_{z}\right)=\{4,27\}, \\
& E\left(3 x^{2}+4 y^{2}+T_{z}\right)=\{2,11,23,50,116,135,138\}, \\
& E\left(x^{2}+2 y^{2}+6 T_{z}\right)=\{5,13,46,161\}, \\
& E\left(x^{2}+5 y^{2}+3 T_{z}\right)=\{2,11,26,37,40,53,62,142,220,425,692\}, \\
& \max E\left(2 x^{2}+2 y^{2}+5 T_{z}\right)=2748, \max E\left(2 x^{2}+4 y^{2}+3 T_{z}\right)=3185, \\
& \max E\left(x^{2}+4 y^{2}+5 T_{z}\right)=2352, \max E\left(2 x^{2}+3 y^{2}+5 T_{z}\right)=933 .
\end{aligned}
$$

Under the generalized Riemann hypothesis, the argument of Ono and Soundararajan [20] would allow one to use Waldspurger's theorem [30] (or a Kohnen-Zagier variant [15] when the corresponding modular form is in Kohnen's plus space) to determine effectively a computationally feasible bound beyond which every integer is represented and hence verify that the above lists (and all lists contained herein) are indeed complete. This is done by carefully comparing the growth of the class numbers of imaginary quadratic fields with the growth of coefficients of a particular cusp form.

Recall that $\left\{x^{2}+2 T_{y}: x, y \in \mathbb{Z}\right\}=\left\{T_{x}+T_{y}: x, y \in \mathbb{Z}\right\}$ as observed by Euler. (See, e.g., (3.6.3) of [1, p.71], and [27, Lemma 1].) Thus we say that $x^{2}+2 T_{y}$ is equivalent to $T_{x}+T_{y}$ and denote this by $x^{2}+2 T_{y} \sim T_{x}+T_{y}$. 
Corollary 1.7. Let $a, b, c \in \mathbb{Z}^{+}$with $c$ odd. Then, all sufficiently large odd integers have the form $2 a x^{2}+2 b y^{2}+c z^{2}$ $\Longleftrightarrow a x^{2}+b y^{2}+4 c T_{z}$ is almost universal $\Longleftrightarrow 2 \| a b,-a b R c,-2 a c R b^{\prime}$ and $-2 b c R a^{\prime}$.

In particular,

all sufficiently large odd numbers are represented by $2 a x^{2}+c\left(y^{2}+z^{2}\right)$

$\Longleftrightarrow a x^{2}+2 c y^{2}+4 c T_{z} \sim a x^{2}+2 c\left(T_{y}+T_{z}\right)$ is almost universal

$\Longleftrightarrow c=1$, and all prime divisors of a are congruent to $1 \bmod 4$.

Remark 1.3. In 2005 L. Panaitopol 21] showed that for $a, b, c \in \mathbb{Z}^{+}$with $a \leq b \leq c$ all positive odd integers can be written in the form $a x^{2}+b y^{2}+c z^{2}$ with $x, y, z \in \mathbb{Z}$, if and only if the vector $(a, b, c)$ is $(1,1,2)$ or $(1,2,3)$ or $(1,2,4)$. For $n \in \mathbb{N}$, clearly $2 n+1=x^{2}+2 y^{2}+3 z^{2}$ for some $x, y, z \in \mathbb{Z}$ if and only if there are $x, y, z \in \mathbb{Z}$ such that $2 n+1=\left(8 T_{x}+1\right)+2 y^{2}+3(2 z)^{2}$ (i.e., $\left.n=4 T_{x}+y^{2}+6 z^{2}\right)$ or $2 n+1=(2 x)^{2}+2 y^{2}+3\left(8 T_{z}+1\right)$ (i.e., $\left.n-1=2 x^{2}+y^{2}+12 T_{z}\right)$. By Corollary 1.7 . the forms $6 x^{2}+y^{2}+4 T_{z}$ and $2 x^{2}+y^{2}+12 T_{z}$ are almost universal. Our computation suggests that

$$
E\left(6 x^{2}+y^{2}+4 T_{z}\right)=\{2,3,17,23,38,51,86,188\}
$$

and

$$
E\left(2 x^{2}+y^{2}+12 T_{z}\right)=\{5,7,10,26,35,65,92,127,322\} .
$$

Corollary 1.8. Let $a, b \in \mathbb{Z}^{+}$with $b$ odd. If $\mathcal{S F}\left(a^{\prime}\right)$ or $\mathcal{S F}(b)$ has a prime divisor $p \equiv 3(\bmod 4)$ (which happens when $a^{\prime}$ or $b$ is congruent to $\left.3 \bmod 4\right)$, then

$$
\begin{aligned}
& a x^{2}+b y^{2}+2 T_{z} \text { is almost universal } \\
\Longleftrightarrow & a x^{2}+y^{2}+2 b T_{z} \text { is almost universal } \\
\Longleftrightarrow & -a R b \text { and }-b R a^{\prime}
\end{aligned}
$$

and

$$
\begin{aligned}
& a x^{2}+2 y^{2}+b T_{z} \text { is almost universal } \\
\Longleftrightarrow & a x^{2}+2 b y^{2}+T_{z} \text { is almost universal } \\
\Longleftrightarrow & -2 a R b \text { and }-b R a^{\prime} .
\end{aligned}
$$

Corollary 1.9. Let a be any positive integer.

(i) The form $a x^{2}+y^{2}+T_{z}$ is almost universal if and only if $-2 R$ a (i.e., every odd prime divisor of a is congruent to 1 or 3 modulo 8). Also, ax $x^{2}+2 y^{2}+2 T_{z}$ is almost universal if and only if each prime divisor of a is congruent to 1 or 3 modulo 8.

(ii)

$$
a x^{2}+2 y^{2}+T_{z} \text { is almost universal }
$$

$\Longleftrightarrow a x^{2}+y^{2}+2 T_{z} \sim a x^{2}+T_{y}+T_{z}$ is almost universal

$\Longleftrightarrow-1 R a^{\prime}$, i.e., every odd prime divisor of a is congruent to $1 \bmod 4$. 
Also,

$$
a x^{2}+2 y^{2}+4 T_{z} \sim a x^{2}+2 T_{y}+2 T_{z} \text { is almost universal }
$$

$\Longleftrightarrow$ all prime divisors of a are congruent to $1 \bmod 4$

and

$a x^{2}+4 y^{2}+2 T_{z}$ is almost universal

$\Longleftrightarrow a \equiv 1(\bmod 8)$ and each prime divisor of a is congruent to $1 \bmod 4$.

Example 1.2. By Corollary [1.9, the form $5 x^{2}+4 y^{2}+2 T_{z}$ is not almost universal, although it is asymptotically universal. Also, our computation suggests the following information:

$$
E\left(11 x^{2}+y^{2}+T_{z}\right)=\{8,34,348\} \text { and } E\left(12 x^{2}+y^{2}+T_{z}\right)=\{8,20,146,275\} .
$$

Corollary 1.10. Let $a \in \mathbb{Z}^{+}$. Then

$a x^{2}+3 y^{2}+T_{z}$ is almost universal (or asymptotically universal)

$\Longleftrightarrow a \equiv 1(\bmod 3)$, and $\lfloor p / 12\rfloor$ is even for any odd prime divisor $p$ of a

and

$a x^{2}+y^{2}+3 T_{z}$ is almost universal (or asymptotically universal)

$\Longleftrightarrow a \equiv 2(\bmod 3)$, and $\lfloor p / 12\rfloor$ is even for any odd prime divisor $p$ of a.

Also,

$a x^{2}+2 y^{2}+6 T_{z}$ is almost universal (or asymptotically universal)

$\Longleftrightarrow a \equiv 1(\bmod 6)$, and $\lfloor p / 12\rfloor$ is even for any prime divisor $p$ of a

and

$a x^{2}+6 y^{2}+2 T_{z}$ is almost universal (or asymptotically universal)

$\Longleftrightarrow a \equiv 5(\bmod 6)$, and $\lfloor p / 12\rfloor$ is even for any prime divisor $p$ of $a$.

Corollary 1.11. Let $m$ be a positive integer.

(i) $x^{2}+y^{2}+m T_{z}$ is almost universal if and only if $4 \nmid m$ and all odd prime divisors of $m$ are congruent to $1 \bmod 4$. Also, $2 x^{2}+y^{2}+m T_{z}$ is almost universal if and only if $8 \nmid m$ and each odd prime divisor of $m$ is congruent to 1 or $3 \bmod 8$.

(ii) Let $k \in \mathbb{Z}^{+}$. Then the form $2^{2 k} x^{2}+y^{2}+m T_{z}$ is almost universal if and only if $4 \nmid m,-1 R m^{\prime}$ and

$$
2 \| m \Longrightarrow m \text { is squarefree. }
$$

Also, the form $2^{2 k+1} x^{2}+y^{2}+m T_{z}$ is almost universal if and only if $4 \nmid m,-2 R m^{\prime}$ and

$$
m \equiv 1(\bmod 8) \Longrightarrow m \text { is squarefree. }
$$

(iii) Let $k, l \in \mathbb{Z}^{+}$with $k \geq l$. Then $2^{k} x^{2}+2^{l} y^{2}+m T_{z}$ is asymptotically universal if and only if for each prime divisor $p$ of $m$ we have

$$
\begin{cases}p \equiv 1(\bmod 4) & \text { if } k \equiv l(\bmod 2), \\ p \equiv 1 \text { or } 3(\bmod 8) & \text { otherwise. }\end{cases}
$$

When $2^{k} x^{2}+2^{l} y^{2}+m T_{z}$ is asymptotically universal, it is almost universal if and only if $m$ is squarefree, or both $2 \mid k$ and $l=1$. 
Example 1.3. By Corollary 1.11 the forms $4 x^{2}+y^{2}+50 T_{z}, 8 x^{2}+y^{2}+9 T_{z}$ and $2 x^{2}+2 y^{2}+25 T_{z}$ are not almost universal, although they are asymptotically universal. We also have the following observations via computation:

$$
\begin{aligned}
& \max E\left(x^{2}+y^{2}+10 T_{z}\right)=546, \max E\left(2 x^{2}+y^{2}+11 T_{z}\right)=985, \\
& \max E\left(4 x^{2}+y^{2}+10 T_{z}\right)=5496, \max E\left(4 x^{2}+2 y^{2}+9 T_{z}\right)=9555, \\
& \max E\left(2 x^{2}+2 y^{2}+13 T_{z}\right)=22176, \max E\left(8 x^{2}+y^{2}+3 T_{z}\right)=499 .
\end{aligned}
$$

Corollary 1.12. Let $a \in \mathbb{Z}^{+}$be even.

(i) Suppose that $v_{2}(a)$ is even and each odd prime divisor of a is congruent to 1 modulo 3. Then $a x^{2}+216 y^{2}+T_{z}$ is asymptotically universal. Moreover, this form is not almost universal if and only if every prime divisor of $\mathcal{S} \mathcal{F}\left(a^{\prime}\right)$ is congruent to 1 or 19 modulo 24, and the number of prime divisors congruent to 19 modulo 24 is odd.

(ii) Assume that $v_{2}(a)$ is odd, $a^{\prime} \equiv \pm 1(\bmod 10)$ and $2 \mid\lfloor p / 10\rfloor$ for every prime divisor $p$ of $a^{\prime}$. Then $a x^{2}+250 y^{2}+T_{z}$ is asymptotically universal. Moreover, this form is not almost universal if and only if $a^{\prime} \equiv 21,29(\bmod 40)$ and every prime divisor of $\mathcal{S F}\left(a^{\prime}\right)$ is congruent to 1 or 9 modulo 20.

Remark 1.4. Corollary 1.12 implies that the forms $76 x^{2}+216 y^{2}+T_{z}$ and $58 x^{2}+$ $250 y^{2}+T_{z}$ are asymptotically universal but not almost universal.

For the form $a x^{2}+b T_{y}+c T_{z}$, we obtain the following result.

Theorem 1.13. Let $a, b, c \in \mathbb{Z}^{+}$with $\operatorname{gcd}(a, b, c)=1$ and $v_{2}(b) \geq v_{2}(c)$. Consider the form

$$
f(x, y, z):=a x^{2}+b T_{y}+c T_{z}
$$

and assume that both (1) and (2) in Theorem 1.2 hold.

(i) When $v_{2}(b) \notin\{3,4\}, f$ is not almost universal if and only if we have the following:

(1) $4 \nmid b+c$ and $\mathcal{S F}\left(a^{\prime} b^{\prime} c^{\prime}\right) \equiv(b+c)^{\prime}\left(\bmod 2^{3-v}\right)$, where $v:=v_{2}(b+c)<2$.

(2) All prime divisors of $\mathcal{S} \mathcal{F}\left(a^{\prime} b^{\prime} c^{\prime}\right)$ are congruent to 1 or 3 modulo 8 if $\mathcal{S} \mathcal{F}(a b c)$ $\equiv b+c(\bmod 2)$ and are congruent to 1 modulo 4 otherwise.

(3) $8 a x^{2}+b y^{2}+c z^{2}=2^{v} \mathcal{S F}\left(a^{\prime} b^{\prime} c^{\prime}\right)$ has no integral solutions with $y$ and $z$ odd.

$$
\left\{\begin{array}{l}
v_{2}(b) \leq 1 \Rightarrow v_{2}(a)-v_{2}(b) \in\{2,4,6, \ldots\} \\
v_{2}(b)=2 \Rightarrow v_{2}(a) \in\{1,3,5, \ldots\} \\
v_{2}(b) \in\{5,7, \ldots\} \Rightarrow(4 \mid a \text { or } 2 \mid c) \\
v_{2}(b) \in\{6,8, \ldots\} \Rightarrow(2 \mid a \text { or } a \equiv c(\bmod 8)) .
\end{array}\right.
$$

(ii) In the case $v_{2}(b) \in\{3,4\}$, if $f$ is not almost universal, then (1) - (3) above hold and also

$$
\left\{\begin{array}{l}
v_{2}(b)=3 \Rightarrow(4 \mid \text { a or } 2 \mid c), \\
v_{2}(b)=4 \Rightarrow(2 \mid \text { a or } a \equiv c(\bmod 8)) .
\end{array}\right.
$$

Moreover, provided (1) - (3) in part (i) and the condition $2 \nmid v_{2}(a), f$ is not almost universal if $v_{2}(b)=4$, or $v_{2}(a) \geq v_{2}(b)=3$ and $b^{\prime} \equiv c^{\prime}(\bmod 8)$.

Example 1.4. Consider those forms $a x^{2}+b T_{y}+c T_{z}$ with $a, b, c \in \mathbb{Z}^{+}$and $a+b+c \leq$ 10. By Theorem 1.13 we find that those asymptotically universal ones are almost 
universal. Below is a complete list of those forms $a x^{2}+b T_{y}+c T_{z}$ with $a, b, c \in \mathbb{Z}^{+}$ and $a+b+c \leq 10$ which are almost universal but not universal:

$$
\begin{aligned}
& 5 x^{2}+T_{y}+T_{z} \sim x^{2}+5 y^{2}+2 T_{z}, 5 x^{2}+2 T_{y}+2 T_{z} \sim 2 x^{2}+5 y^{2}+4 T_{z}, \\
& 8 x^{2}+T_{y}+T_{z} \sim x^{2}+8 y^{2}+2 T_{z}, 2 x^{2}+3 T_{y}+2 T_{z}, x^{2}+4 T_{y}+3 T_{z}, \\
& 2 x^{2}+5 T_{y}+T_{z}, 4 x^{2}+3 T_{y}+T_{z}, 3 x^{2}+5 T_{y}+T_{z}, 3 x^{2}+4 T_{y}+2 T_{z}, \\
& 4 x^{2}+4 T_{y}+T_{z}, 6 x^{2}+2 T_{y}+T_{z}, 5 x^{2}+3 T_{y}+2 T_{z}, 5 x^{2}+4 T_{y}+T_{z} .
\end{aligned}
$$

For the above forms from the second line, our computation via computer suggests the following information:

$$
\begin{aligned}
& E\left(8 x^{2}+T_{y}+T_{z}\right)=E\left(x^{2}+8 y^{2}+2 T_{z}\right)=\{5,40,217\}, \\
& E\left(2 x^{2}+3 T_{y}+2 T_{z}\right)=\{1,16\}, E\left(x^{2}+4 T_{y}+3 T_{z}\right)=\{2,6,80\}, \\
& E\left(2 x^{2}+5 T_{y}+T_{z}\right)=\{4\}, E\left(4 x^{2}+3 T_{y}+T_{z}\right)=\{2,11,27,38,86,93,188,323\}, \\
& E\left(3 x^{2}+5 T_{y}+T_{z}\right)=\{2,7\}, E\left(3 x^{2}+4 T_{y}+2 T_{z}\right)=\{1,8,11,25\}, \\
& E\left(4 x^{2}+4 T_{y}+T_{z}\right)=\{2,108\}, E\left(6 x^{2}+2 T_{y}+T_{z}\right)=\{4\}, \\
& E\left(5 x^{2}+3 T_{y}+2 T_{z}\right)=\{1,4,13,19,27,46,73,97,111,123,151,168\}, \\
& E\left(5 x^{2}+4 T_{y}+T_{z}\right)=\{2,16,31\} .
\end{aligned}
$$

In Corollary 1.9 we determined when $a x^{2}+T_{x}+T_{y}$ or $a x^{2}+2 T_{x}+2 T_{y}$ is almost universal. The following corollary deals with two other similar forms.

Corollary 1.14. Let a be a positive integer. Then a $x^{2}+2 T_{y}+T_{z}$ is almost universal if and only if all odd prime divisors of a are congruent to 1 or $3 \bmod 8$. Also, $a x^{2}+4 T_{y}+T_{z}$ is almost universal if and only if all odd prime divisors of a are congruent to $1 \bmod 4$.

Example 1.5. By means of computation, we believe that

$$
E\left(9 x^{2}+2 T_{y}+T_{z}\right)=\{4\} \text { and } E\left(11 x^{2}+2 T_{y}+T_{z}\right)=\{4,25,94,123\} .
$$

Corollary 1.15. Let $m$ be any positive integer.

(i) If all odd prime divisors of $m$ are congruent to 1 or $3 \bmod 8$, and $m^{\prime} \equiv$ $3(\bmod 8)$ or $v_{2}(m) \neq 4,6, \ldots$, then $x^{2}+T_{y}+m T_{z}$ is almost universal. The converse also holds when $v_{2}(m) \neq 4$.

(ii) For $k \in \mathbb{Z}^{+} \backslash\{3,4\}$, the form $2^{k}\left(x^{2}+T_{y}\right)+m T_{z}$ is almost universal if and only if $k \in\{1,2\}$ and all prime divisors of $m$ are congruent to 1 or $3 \bmod 8$. When $m \equiv 1(\bmod 8)$, the form $8\left(x^{2}+T_{y}\right)+m T_{z}$ is not almost universal.

Example 1.6. By Corollary 1.15, the form $8 x^{2}+8 T_{y}+T_{z}$ is not almost universal, although it is asymptotically universal. We also have the following guess based on our computation:

$E\left(x^{2}+T_{y}+9 T_{z}\right)=\{8,47\}, E\left(x^{2}+T_{y}+11 T_{z}\right)=\{8\}, E\left(x^{2}+T_{y}+12 T_{z}\right)=\{8,20\}$.

Corollary 1.16. Let $m$ be any positive integer.

(i) When $v_{2}(m) \neq 3$, the form $x^{2}+2 T_{y}+m T_{z} \sim T_{x}+T_{y}+m T_{z}$ is almost universal if and only if all odd prime divisors of $m$ are congruent to $1 \bmod 4$ and $v_{2}(m) \neq 5,7, \ldots$

(ii) For $k \in \mathbb{Z}^{+} \backslash\{2\}$, the form $2^{k}\left(x^{2}+2 T_{y}\right)+m T_{z} \sim 2^{k}\left(T_{x}+T_{y}\right)+m T_{z}$ is almost universal if and only if $k=1$ and all prime divisors of $m$ are congruent to $1 \bmod 4$. 
Remark 1.5. By Corollary 1.16(ii), $8 x^{2}+16 T_{y}+T_{z} \sim 8\left(T_{x}+T_{y}\right)+T_{z}$ is not almost universal, although it is asymptotically universal. In [29] the second author conjectured that any integer $n>1029$ is either a triangular number or a sum of two odd squares and a triangular number (i.e., $n=\left(8 T_{x}+1\right)+\left(8 T_{y}+1\right)+T_{z}$ for some $x, y, z \in \mathbb{Z})$; in other words,

$$
E\left(8 T_{x}+8 T_{y}+T_{z}\right) \cap[1028,+\infty) \subseteq\left\{T_{m}-2: m \in \mathbb{Z}^{+}\right\} .
$$

Recently, Oh and the second author [16] showed that $T_{m}-2 \in E\left(8 T_{x}+8 T_{y}+T_{z}\right)$ (i.e., $T_{m}$ is not a sum of two odd squares and a triangular number) if and only if $2 m+1$ is a prime congruent to $3 \bmod 4$.

Example 1.7. Via computation we make the following observation:

$$
\begin{aligned}
& E\left(x^{2}+2 T_{y}+10 T_{z}\right)=E\left(T_{x}+T_{y}+10 T_{z}\right)=\{5,8\}, \\
& E\left(x^{2}+2 T_{y}+13 T_{z}\right)=E\left(T_{x}+T_{y}+13 T_{z}\right)=\{5,8,32,53\} .
\end{aligned}
$$

Theorem 1.17. Let $a, b, c \in \mathbb{Z}^{+}$with $v_{2}(a) \geq v_{2}(b) \geq v_{2}(c)=0$. Assume that -bc $R a^{\prime},-a c R b^{\prime}$ and -ab $R c^{\prime}$. Consider the form

$$
f(x, y, z):=a T_{x}+b T_{y}+c T_{z} .
$$

(i) If $f$ is not almost universal, then we have the following:

(1) $4 \nmid a+b+c$ and $\mathcal{S F}\left(a^{\prime} b^{\prime} c^{\prime}\right) \equiv(a+b+c)^{\prime}\left(\bmod 2^{3-v}\right)$, where $v=$ $v_{2}(a+b+c)<2$.

(2) All prime divisors of $\mathcal{S} \mathcal{F}\left(a^{\prime} b^{\prime} c^{\prime}\right)$ are congruent to 1 modulo 4 if $\mathcal{S} \mathcal{F}(a b c) \equiv$ $a+b+c(\bmod 2)$ and are congruent to 1 or 3 modulo 8 otherwise.

(3) $a x^{2}+b y^{2}+c z^{2}=2^{v} \mathcal{S F}\left(a^{\prime} b^{\prime} c^{\prime}\right)$ has no integral solution with $x, y, z$ all odd.

$$
\left\{\begin{array}{l}
v_{2}(b) \leq 1 \Rightarrow v_{2}(a)-v_{2}(b) \in\{3,5,7, \ldots\}, \\
v_{2}(b)=2 \Rightarrow v_{2}(a) \in\{2,4,6, \ldots\} .
\end{array}\right.
$$

(ii) $f$ is not almost universal under (11)-(3) in part (i), and the following condition stronger than (4):

$$
\left\{\begin{array}{l}
v_{2}(b) \leq 1 \Rightarrow v_{2}(a)-v_{2}(b) \in\{5,7, \ldots\} \\
v_{2}(b) \in\{2,4\} \Rightarrow v_{2}(a) \in\{4,6, \ldots\} \\
v_{2}(b)=3 \Rightarrow\left(v_{2}(a) \in\{6,8, \ldots\} \& b^{\prime} \equiv c(\bmod 8)\right) .
\end{array}\right.
$$

Example 1.8. Consider those forms $a T_{x}+b T_{y}+c T_{z}$ with $a, b, c \in \mathbb{Z}^{+}$and $a+b+c \leq$ 10. By Theorem [1.3. we find the following complete list of those asymptotically universal forms which are not universal:

$$
\begin{aligned}
& T_{x}+4 T_{y}+4 T_{z} \sim 4 x^{2}+8 T_{y}+T_{z}, \\
& T_{x}+T_{y}+8 T_{z} \sim x^{2}+8 T_{y}+2 T_{z}, \\
& 2 T_{x}+2 T_{y}+5 T_{z} \sim 2 x^{2}+4 T_{y}+5 T_{z}, \\
& T_{x}+2 T_{y}+6 T_{z}, 2 T_{x}+3 T_{y}+4 T_{z}, T_{x}+4 T_{y}+5 T_{z} .
\end{aligned}
$$

By Theorem 1.17 the last four forms are in fact almost universal; our computation via computer suggests the following information:

$E\left(2 T_{x}+2 T_{y}+5 T_{z}\right)=E\left(2 x^{2}+4 T_{y}+5 T_{z}\right)=\{1,3,10,16,28,43,46,85,169,175,211,223\}$ 
and

$E\left(T_{x}+2 T_{y}+6 T_{z}\right)=\{4,50\}, E\left(2 T_{x}+3 T_{y}+4 T_{z}\right)=\{1,8,31\}, E\left(T_{x}+4 T_{y}+5 T_{z}\right)=\{2\}$.

As for the first two forms $T_{x}+4 T_{y}+4 T_{z}$ and $T_{x}+T_{y}+8 T_{z}$, neither Theorem 1.17 nor Theorem 1.13 tells us whether or not they are almost universal. However, with some special arguments, the first author [12] was able to show that they are not almost universal, although they are asymptotically universal. By Theorem 1.1(ii) of an earlier paper [16], $E\left(T_{x}+T_{y}+8 T_{z}\right)$ actually consists of those $2 T_{m}-1$ $\left(m \in \mathbb{Z}^{+}\right.$) with $2 m+1$ having no prime divisors congruent to $3 \bmod 4$. Similarly, by [27, Theorem 1(iii)] and [16, Theorem 2.1(ii)], $E\left(T_{x}+4 T_{y}+4 T_{z}\right)$ consists of those $T_{m}-1\left(m \in \mathbb{Z}^{+}\right)$with $2 m+1$ having no prime divisors congruent to $3 \bmod$ 4.

Corollary 1.18. Let $a \in \mathbb{Z}^{+}$. Then the form $a T_{x}+2 T_{y}+T_{z}$ is almost universal if each odd prime divisor of a is congruent to 1 or $3 \bmod 8$, and either $a^{\prime} \equiv 1(\bmod 8)$ or $v_{2}(a) \neq 4,6, \ldots$. We also have the converse when $v_{2}(a) \neq 4$.

Remark 1.6. In [12] the first author was able to show that the special form $48 T_{x}+$ $2 T_{y}+T_{z}$ is not almost universal (though it is asymptotically universal by Theorem 1.3 .

Example 1.9. Our computation leads us to make the following observation:

$$
\begin{gathered}
E\left(9 T_{x}+2 T_{y}+T_{z}\right)=\{4,46\}, E\left(11 T_{x}+2 T_{y}+T_{z}\right)=\{4,25\}, \\
E\left(22 T_{x}+2 T_{y}+T_{z}\right)=\{4,11,14,19,46,54\} .
\end{gathered}
$$

Our following conjecture is a supplement to Theorems 1.13 and 1.17, its solution might involve a further investigation of the spinor norm mapping or alternation of certain coefficients of cusp forms.

Conjecture 1.19. Let $a, b, c$ be positive integers.

(i) In the case $v_{2}(b) \geq v_{2}(c)$ and $v_{2}(b) \in\{3,4\}$, if $(1)-(3)$ in Theorem 1.13 hold and also

$$
\left\{\begin{array}{l}
v_{2}(b)=3 \Rightarrow(4 \mid a \text { or } 2 \mid c), \\
v_{2}(b)=4 \Rightarrow(2 \mid a \text { or } a \equiv c(\bmod 8)),
\end{array}\right.
$$

then the form $a x^{2}+b T_{y}+c T_{z}$ is not almost universal.

(ii) In the case $v_{2}(a) \geq v_{2}(b) \geq v_{2}(c)=0$, if (1) - (3) in Theorem 1.17 hold and

$$
v_{2}(a)=v_{2}(b)=2 \quad \text { or } \quad v_{2}(a)=v_{2}(b)+3 \in\{3,4\} \quad \text { or } \quad v_{2}(b) \in\{3,4\},
$$

then the form $a T_{x}+b T_{y}+c T_{z}$ is not almost universal.

In the next section we are going to introduce some further notation and give an overview of our method. In Section 3 we will deal with asymptotically universal forms and prove Theorems 1.1-1.3 and Corollaries 1.4-1.5. Section 4 is devoted to the proofs of the remaining theorems and corollaries concerning almost universal forms.

\section{Notation AND BRIEF OVERVIEW}

Our arguments will involve the theory of modular forms and spinor exceptional square classes for quadratic forms. A good introduction to modular forms may be found in Ono's book [18, and a good introduction to quadratic forms may be found in O'Meara's book [17]. We will first reduce the questions at hand to 
questions about certain related (ternary) quadratic forms. Since $8 T_{x}+1$ is an odd square, multiplying by 8 and adding some positive integer will give a form $Q(x, y, z)$ which is a sum of squares with the restriction that some of $x, y$, and $z$ must be odd. If we take $r_{Q}(n)$ to be the number of solutions to $Q(x, y, z)=n$ with the given restrictions on $x, y, z \in \mathbb{Z}$, then define

$$
\theta_{Q}(\tau):=\sum_{n=0}^{\infty} r_{Q}(n) q^{n},
$$

where $q=e^{2 \pi i \tau}$ with $\tau$ in the upper half plane. Since the number of solutions with $z$ odd equals the number of solutions with $z$ arbitrary minus the number of solutions with $z$ even and since the form with $z$ even gives another quadratic form, we get an inclusion/exclusion of theta series of quadratic forms. Let a ternary quadratic form $Q^{\prime}(x, y, z)$ be given. Then it is well known that the theta series

$$
\theta_{Q^{\prime}}(\tau):=\sum_{n=0}^{\infty} r_{Q^{\prime}}(n) q^{n}
$$

is a modular form of weight $3 / 2$, where $r_{Q^{\prime}}(n)$ is the number of solutions to $Q^{\prime}(x, y, z)=n$ with $x, y, z \in \mathbb{Z}$. The theta series splits naturally into the following three parts:

$$
\theta_{Q^{\prime}}=\theta_{\text {gen }\left(Q^{\prime}\right)}+\left(\theta_{\operatorname{spn}\left(Q^{\prime}\right)}-\theta_{g e n\left(Q^{\prime}\right)}\right)+\left(\theta_{Q^{\prime}}-\theta_{\operatorname{spn}\left(Q^{\prime}\right)}\right),
$$

where the $n$-th coefficients of $\theta_{\operatorname{gen}\left(Q^{\prime}\right)}$ and $\theta_{\operatorname{spn}\left(Q^{\prime}\right)}$ are the weighted average of the number of representations of $n$ by the genus and the spinor genus of $Q^{\prime}$, respectively. Furthermore, $\theta_{g e n}\left(Q^{\prime}\right)$ is an Eisenstein series, $\theta_{\operatorname{spn}\left(Q^{\prime}\right)}-\theta_{g e n\left(Q^{\prime}\right)}$ is a cusp form in the space of 1-dimensional theta series, and $\theta_{Q^{\prime}}-\theta_{\operatorname{spn}\left(Q^{\prime}\right)}$ is a cusp form in the orthogonal complement of the space of 1-dimensional theta series. For a full description, see the survey paper of Schulze-Pillot [23]. We will then use the argument of Duke and Schulze-Pillot [6].

The coefficients of $\theta_{\operatorname{spn}\left(Q^{\prime}\right)}-\theta_{\text {gen }\left(Q^{\prime}\right)}$ are supported at finitely many square classes. If $r_{Q^{\prime}, p^{k}}(n)$ is the number of solutions to $Q^{\prime}(x, y, z)=n+p^{k} \mathbb{Z}$ with $x, y, z \in \mathbb{Z} / p^{k} \mathbb{Z}$, then the $n$-th coefficient of the Eisenstein series was shown by Siegel (cf. Jones [1]) to be

$$
\prod_{p \text { prime }} \lim _{k \rightarrow \infty} \frac{r_{Q^{\prime}, p^{k}}(n)}{p^{2 k}} .
$$

An anisotropic prime $p$ is a prime for which the equation $Q^{\prime}(x, y, z)=0$ has no non-trivial solutions in $\mathbb{Z}_{p}$. Notice that for $q \neq p, r_{Q^{\prime}, q^{k}}\left(n p^{2}\right)=r_{Q^{\prime}, q^{k}}(n)$, since $p$ is invertible, and hence we have a bijection between solutions to $Q^{\prime}(x, y, z)=n p^{2}$ and $Q^{\prime}\left(x^{\prime}, y^{\prime}, z^{\prime}\right)=n$ by taking $(x, y, z) \rightarrow p^{-1}(x, y, z)$. Because $Q^{\prime}(x, y, z)=0$, if $n$ has sufficiently large divisibility by $p$ (i.e., the order of $n$ at $p$ is large enough) then it is easy to check that $r_{Q^{\prime}, p^{k}}\left(n p^{2}\right)=r_{Q^{\prime}, p^{k}}(n)$, and hence the $n p^{2 k}$ coefficients of the Eisenstein series grow like a constant with respect to $k$.

When $n$ has bounded divisibility at every anisotropic prime (i.e., the orders of $n$ at anisotropic primes are bounded) equation (2.1), and hence the coefficients of the Eisenstein series, grow like a certain class number (cf. Jones [11, Theorem 86]), and hence are (ineffectively) $\gg n^{1 / 2-\epsilon}$ by the bound of Siegel [26]. The coefficients of the cusp forms in the orthogonal complement of 1-dimensional theta series (to which $\theta_{Q^{\prime}}-\theta_{\operatorname{spn}\left(Q^{\prime}\right)}$ belongs) were $\ll n^{1 / 2-1 / 28+\epsilon}$ as first obtained by Duke [5], extending 
Iwaniec's result [10] (for coefficients of half integral weight $\geq 5 / 2$ modular forms) to the case of weight $3 / 2$ modular forms. Better bounds have been obtained by the amplification method on sums of special values of $L$-series as in Blomer, Harcos, and Michel [2, but the bound above is sufficient to guarantee that if $n$ has bounded divisibility at the anisotropic primes and $n$ is not in one of the finitely many square classes where the coefficients of $\theta_{\operatorname{spn}\left(Q^{\prime}\right)}-\theta_{\operatorname{gen}\left(Q^{\prime}\right)}$ are supported, the growth of the coefficients of the Eisenstein series $\theta_{\text {gen }\left(Q^{\prime}\right)}$ will overwhelm the growth of the coefficients of the cusp form $\theta_{Q^{\prime}}-\theta_{\text {gen }\left(Q^{\prime}\right)}$, and hence the coefficients of $\theta_{Q^{\prime}}$ will be positive for sufficiently large $n$, with bounded divisibility by the anisotropic primes, outside of these finitely many square classes. Moreover, if we take a weighted sum

$$
\sum_{i=1}^{m} w_{i} \theta_{Q_{i}^{\prime}}
$$

(such as the inclusion/exclusion above) of finitely many such $\theta_{Q_{i}^{\prime}}$ where $\theta_{\text {gen }\left(Q_{i}^{\prime}\right)}=$ $c_{i} \theta_{\text {gen }\left(Q^{\prime}\right)}$ and $\sum_{i=1}^{m} w_{i} c_{i}>0$, then the resulting theta series will be

$$
\left(\sum_{i=1}^{m} w_{i} c_{i}\right) \theta_{g e n\left(Q^{\prime}\right)}+f_{1}+f_{2},
$$

where $f_{1}=\sum_{i=1}^{m} w_{i}\left(\theta_{\operatorname{spn}\left(Q_{i}^{\prime}\right)}-\theta_{\text {gen }\left(Q_{i}^{\prime}\right)}\right)$ and $f_{2}=\sum_{i=1}^{m} w_{i}\left(\theta_{Q_{i}^{\prime}}-\theta_{s p n}\left(Q_{i}^{\prime}\right)\right)$. The bound of Duke [5] given above then shows that outside of the finitely many square classes where the coefficients of $f_{1}$ are supported, the $n$-th coefficient of this weighted average is positive for sufficiently large $n$ with bounded divisibility at the anisotropic primes. The condition of the bounded divisibility at anisotropic primes will pose only a minor complication, and we will find in the end that for any asymptotically universal form the associated quadratic forms will never have an anisotropic prime $p \neq 2$, while conditions modulo 8 will guarantee that the coefficients which we are interested in automatically have bounded divisibility by 2 .

We will thus be interested in determining which square classes of coefficients $t \mathbb{Z}^{2}$ are supported by $\theta_{\operatorname{spn}\left(Q^{\prime}\right)}-\theta_{\text {gen }\left(Q^{\prime}\right)}$. Kneser [14] gave a necessary condition and later Schulze-Pillot [24] extended this to give necessary and sufficient conditions. For a quadratic form $Q^{\prime}$, there is an associated bilinear form $B(x, y)=$ $\left(Q^{\prime}(x+y)-Q^{\prime}(x)-Q^{\prime}(y)\right) / 2$. We will call $V$ a (ternary) quadratic space over $\mathbb{Q}_{2}$ if it is a finite-dimensional vector space over $\mathbb{Q}_{2}$ with an associated bilinear form $B$. There is a quadratic form (over $\mathbb{Q}_{2}$ ) associated to $V$ given by $Q^{\prime}(x)=B(x, x)$ for every $x \in V$. Fix a $\mathbb{Z}_{2}$-lattice $L$. The quadratic form (over $\mathbb{Z}_{2}$ ) associated to $L$ is $Q^{\prime}(x)=B(x, x)$ with $x \in L$. In our case, the lattice will always have an orthogonal basis $x_{1}, x_{2}, x_{3}$ with $B\left(x_{i}, x_{j}\right)=0$ when $i \neq j$. We will denote the $\mathbb{Z}_{2}$-lattice whose corresponding quadratic form is $a x^{2}+b y^{2}+c z^{2}$ by $\langle a, b, c\rangle_{2}$.

We will denote isometries from $V$ to $V$ by $O(V)$. Let $O^{+}(V)$ be the subgroup of rotations consisting of isometries with determinant 1 . We also use $O^{+}(L)$ to denote the rotations which fix $L$. Each rotation is the product of an even number of symmetries, where the symmetry $\tau_{v}$ with $v \in V$ is defined by

$$
x \mapsto x-\frac{2 B(x, v)}{Q^{\prime}(v)} v .
$$

The spinor norm mapping is the mapping $\theta(\sigma)=Q^{\prime}\left(v_{1}\right) \cdots Q^{\prime}\left(v_{m}\right) \mathbb{Q}_{2}^{\times^{2}}$ where $\sigma=$ $\tau_{v_{1}} \cdots \tau_{v_{m}}$. The set $\theta\left(O^{+}(L)\right)$ forms a subgroup of $\mathbb{Q}_{2}^{\times} / \mathbb{Q}_{2}^{\times 2}$. For the 2-adic lattice $L=L_{2}=\langle a, b, c\rangle_{2}$, Earnest and Hsia determined this subgroup explicitly in [7]. 
Fix an imaginary quadratic field extension $K / \mathbb{Q}$ (in our cases, $K=\mathbb{Q}(i)$ or $K=\mathbb{Q}(\sqrt{-2})$ ) and a prime ideal $\beta$ (of the ring $O_{K}$ of algebraic integers in $K$ ) dividing 2. For convenience, we define

$$
K_{n}:=\mathbb{Q}(\sqrt{-n \mathcal{S F}(n)}) .
$$

We say that $\alpha \in \mathbb{Q}_{2}^{\times} / \mathbb{Q}_{2}^{\times 2}$ is a local norm at 2 (from the completion $K_{\beta}$ to $\mathbb{Q}_{2}$ ) if $\alpha=x^{2}+n y^{2}$ for some $x, y \in \mathbb{Q}_{2}$. We will denote the set of local norms at 2 by $N_{2}(K)$. Note that

$$
N_{2}(\mathbb{Q}(i))=\mathbb{Q}_{2}^{\times 2} \cup 5 \mathbb{Q}_{2}^{\times^{2}} \cup 2 \mathbb{Q}_{2}^{\times 2} \cup 10 \mathbb{Q}_{2}^{\times^{2}}
$$

and

$$
N_{2}(\mathbb{Q}(\sqrt{-2}))=\mathbb{Q}_{2}^{\times 2} \cup 3 \mathbb{Q}_{2}^{\times 2} \cup 2 \mathbb{Q}_{2}^{\times^{2}} \cup 6 \mathbb{Q}_{2}^{\times^{2}}
$$

Using explicit results of Earnest, Hsia, and Hung 8 based on Schulze-Pillot's classification of spinor exceptional square classes 24, we will reduce the question at hand to showing Kneser's necessary condition at the prime 2. The necessary condition of Kneser which we will need to show is that $\theta\left(O^{+}(L)\right) \subseteq N_{2}(K)$ (cf. [14). We will use the explicit results of Earnest and Hsia [7] to determine when the necessary condition is satisfied.

For $a, b \in \mathbb{Q}_{2}^{\times}$, the Hilbert symbol $(a, b)_{2} \in\{ \pm 1\}$ takes the value 1 if and only if $a x^{2}+b y^{2}=z^{2}$ for some $x, y, z \in \mathbb{Q}_{2}$ with $x, y, z$ not all zero. We will need the following theorems.

Theorem 2.1 (Earnest and Hsia [7]). Let $U$ denote the group of units in $\mathbb{Z}_{2}$ and let $\alpha \in U$. Then

$$
\theta\left(O^{+}\left(\left\langle 1,2^{r} \alpha\right\rangle_{2}\right)\right)= \begin{cases}\left\{\gamma \in \mathbb{Q}_{2}^{\times}:(\gamma,-2 \alpha)_{2}=1\right\} & \text { if } r \in\{1,3\}, \\ \left\{\gamma \in U \mathbb{Q}_{2}^{\times^{2}}:(\gamma,-\alpha)_{2}=1\right\} & \text { if } r=2, \\ \mathbb{Q}_{2}^{\times^{2}} \cup \alpha \mathbb{Q}_{2}^{\times^{2}} \cup 5 \mathbb{Q}_{2}^{\times^{2}} \cup 5 \alpha \mathbb{Q}_{2}^{\times 2} & \text { if } r=4, \\ \mathbb{Q}_{2}^{\times^{2}} \cup \alpha \mathbb{Q}_{2}^{\times^{2}} & \text { if } r \geq 5 .\end{cases}
$$

Furthermore, Earnest and Hsia [7, Theorem 2.2] showed that for the lattice $L_{2}:=\left\langle c^{\prime}, 2^{r} b^{\prime}, 2^{s} a^{\prime}\right\rangle_{2}$, we have $\theta\left(O^{+}\left(L_{2}\right)\right)=\mathbb{Q}_{2}^{\times}$if $\{r, s-r\} \cap\{1,3\} \neq \emptyset$ and $\{r, s, s-r\} \cap\{2,4\} \neq \emptyset$. If $0<r<s$ and the conditions of Theorem 2.2 in [7] are not satisfied, they proved that $\theta\left(O^{+}\left(L_{2}\right)\right)$ is equal to the union of the spinor norm restricted to 2-dimensional sublattices, allowing us to use the above theorem. Moreover, if $s \geq 5$ and $r \in\{0, s\}$, then their argument follows mutatis mutandis and will also allow us to reduce the problem to 2 -dimensional sublattices.

Since our base field is $\mathbb{Q}_{2}$ and $K_{\beta} / \mathbb{Q}_{2}$ is ramified for $K=\mathbb{Q}(i)$ and $K=\mathbb{Q}(\sqrt{-2})$, we will only need the following restriction of the 2-adic conditions from Earnest, Hsia, and Hung's theorem.

Theorem 2.2 (Earnest, Hsia, and Hung [8]). Let $a, b, c \in \mathbb{Z}^{+}$and $K=\mathbb{Q}(\sqrt{-a b c})$. Let $L_{2}=\left\langle c^{\prime}, 2^{r} b^{\prime}, 2^{s} a^{\prime}\right\rangle_{2}$ with $0 \leq r \leq s$, and let $t \in \mathbb{Z}^{+}$. Assume that $\theta\left(O^{+}\left(L_{2}\right)\right) \subseteq$ $\mathrm{N}_{2}(K)$, and define

$$
L^{\prime}= \begin{cases}\left\langle 2^{r-2} c^{\prime}, 2^{r} b^{\prime}, 2^{s} a^{\prime}\right\rangle_{2} & \text { if } r+s \equiv v_{2}(t)(\bmod 2), \\ \left\langle 2^{r-3} c^{\prime}, 2^{r} b^{\prime}, 2^{s} a^{\prime}\right\rangle_{2} & \text { otherwise }\end{cases}
$$

Consider the necessary and sufficient conditions given by Schulze-Pillot 24] for $t$ to be a primitive spinor exception for the genus of the quadratic form $Q(x, y, z)=$ $a x^{2}+b y^{2}+c z^{2}$. 
(1) Set $L^{\prime \prime}:=\left\langle 2^{r} c^{\prime}, 2^{r} b^{\prime}, 2^{s} a^{\prime}\right\rangle_{2}$. When $r+s \equiv v_{2}(t)(\bmod 2)$, the Schulze-Pillot conditions are not satisfied if and only if one of the following holds:

(a) $r$ is odd and $v_{2}(t) \geq r-3$.

(b) $r$ is even, $\theta\left(O^{+}\left(L^{\prime}\right)\right) \nsubseteq N_{2}(K)$, and

$$
\left(r \neq s \& v_{2}(t) \geq r-2\right) \text { or }\left(r=s \& v_{2}(t) \geq r\right) .
$$

(c) $r$ is even, $\theta\left(O^{+}\left(L^{\prime}\right)\right) \subseteq N_{2}(K), \theta\left(O^{+}\left(L^{\prime \prime}\right)\right) \nsubseteq N_{2}(K)$ and $v_{2}(t) \geq r$.

(d) $r$ is even, $\theta\left(O^{+}\left(L^{\prime}\right)\right) \subseteq N_{2}(K), \theta\left(O^{+}\left(L^{\prime \prime}\right)\right) \subseteq N_{2}(K)$ and $v_{2}(t) \geq s$.

(2) When $r+s \not \equiv v_{2}(t)(\bmod 2)$, we have $0<r<s$, and the Schulze-Pillot conditions are not satisfied if and only if one of the following holds:

(a) $r$ is even and $v_{2}(t) \geq r-4$.

(b) $r$ is odd, $\theta\left(O^{+}\left(L^{\prime}\right)\right) \nsubseteq N_{2}(K)$ and $v_{2}(t) \geq r-3$.

(c) $r$ is odd, $\theta\left(O^{+}\left(L^{\prime}\right)\right) \subseteq N_{2}(K)$ and $v_{2}(t) \geq s-2$.

\section{ON ASYMPTOTICALLY UNIVERSAL FORMS}

In this section, we will show which forms are asymptotically universal, proving Theorems 1.1, 1.2, and 1.3. We will first need the following lemma.

Lemma 3.1. Fix $a, b, c \in \mathbb{Z}^{+}$with $\operatorname{gcd}(a, b, c)=1$. Then

$$
Q(x, y, z)=a x^{2}+b y^{2}+c z^{2}
$$

represents every integer $p$-adically for each odd prime $p$ if and only if we have

$$
-a b R c^{\prime},-a c R b^{\prime} \text {, and }-b c R a^{\prime} .
$$

Proof. It is well known that $Q$ represents every integer $p$-adically for any odd prime $p$ not dividing $a b c$.

Let $p$ be an odd prime divisor of $a b c$. Without loss of generality, we assume that $p \mid c$. If $p \mid a b$, then $Q$ clearly only represents all squares or all non-squares modulo $p$. Therefore, $p \nmid a b$.

Let a unit $u \in \mathbb{Z}_{p}$ be given. Suppose that there are $x, y \in \mathbb{Z}_{p}$ such that $a x^{2}+b y^{2}=$ $p u$. If $x \in p \mathbb{Z}_{p}$, then we have $y \in p \mathbb{Z}_{p}$ and hence $u \in p \mathbb{Z}_{p}$, which contradicts the fact that $u$ is a unit. Therefore both $x$ and $y$ must be units. Taking the Legendre symbol of both sides yields that

$$
\left(\frac{b}{p}\right)=\left(\frac{b y^{2}}{p}\right)=\left(\frac{p u-a x^{2}}{p}\right)=\left(\frac{-a x^{2}}{p}\right)=\left(\frac{-a}{p}\right) .
$$

Now assume that $\left(\frac{-a b}{p}\right) \neq 1$. Let a unit $u \in \mathbb{Z}_{p}$ be given. From the above, we know that $a x^{2}+b y^{2}=u p$ does not have a solution. Suppose that there are $x, y, z \in \mathbb{Z}_{p}$ such that $a x^{2}+b y^{2}+c z^{2}=u p$. Then

$$
a x^{2}+b y^{2}=\left(u-\frac{c}{p} z^{2}\right) p .
$$

If $p^{2} \mid c$, then $u-\frac{c}{p} z^{2}$ is also a unit, and it follows that up is not represented. In the case $p \| c$, without loss of generality we assume that the unit $\frac{c}{p}$ is a square. If $u$ is not a square, then $u-\frac{c}{p} z^{2}$ must also be a unit, and it follows that $u p$ is not represented.

By the above, (3.1) is a necessary condition.

If $n \in \mathbb{Z}_{p}$ is represented, then so is $n p^{2}$. Thus we only need to show that $\left(\frac{-a b}{p}\right)=1$ implies that those $n \in \mathbb{Z}_{p}$ with $v_{p}(n) \in\{0,1\}$ are $p$-adically represented. 
We have already shown that $\left(\frac{-a b}{p}\right)=1$ if and only if those $n \in \mathbb{Z}_{p}$ with $v_{p}(n)=1$ are represented, so we only need to prove that every unit is represented. Hence, it suffices to show that at least one square and one non-square are represented by $Q$. We will prove that $a x^{2}+b y^{2}$ represents every integer $p$-adically. If -1 is not a square, then

$$
\left(\frac{b}{p}\right)=-\left(\frac{a}{p}\right)
$$

and hence both squares and non-squares are represented. So we may assume that -1 is a square. For any unit $u \in \mathbb{Z}_{p}$, the form $a x^{2}+b y^{2}$ represents every integer $p$-adically if and only if $u a x^{2}+u b y^{2}$ represents every integer $p$-adically. So, without loss of generality we may suppose that

$$
\left(\frac{a}{p}\right)=\left(\frac{b}{p}\right)=1
$$

Since -1 is a square and we represent all squares by $a x^{2}$ (and also $b y^{2}$ ), we must represent -1 . We now argue inductively by noting that if $-m$ is a square, then $-m-1$ is represented by $a x^{2}+b y^{2}$ via taking $a x^{2}=-m$ and $b y^{2}=-1$. If $-m-1$ is a non-square, then we are done; if $-m-1$ is a square, then we can continue the induction. Hence we must also represent a non-square, and the proof is concluded.

We are now ready to prove Theorems 1.1, 1.2. and 1.3.

Proof of Theorem 1.1. Since $8 T_{x}+1=(2 x+1)^{2}$, the representation of $n$ by $f(x, y, z)$

$=a x^{2}+b y^{2}+c T_{z}$ is equivalent to the representation of $8 n+c$ by

$$
Q(x, y, z)=8 a x^{2}+8 b y^{2}+c z^{2}
$$

with $z$ odd. The number of the latter representations equals the number of solutions with $z$ arbitrary minus those with $z$ even. As described in Section 2 , every sufficiently large integer locally represented with bounded divisibility at the (finitely many) anisotropic primes of $Q^{\prime}=Q$ or $Q^{\prime}(x, y, 2 z)$ are represented, outside of the finitely many spinor exceptional square classes for $Q^{\prime}(x, y, z)$ or $Q^{\prime}(x, y, 2 z)$. Thus, if the local conditions are satisfied, then

$$
\{8 n+c: n \in E(f)\} \subseteq\left(\bigcup_{j=1}^{r} \bigcup_{p}\left\{n_{j} p^{2 s}: s \in \mathbb{N}\right\}\right) \cup\left(\bigcup_{i=1}^{m} t_{i} \mathbb{Z}^{2}\right),
$$

where $p$ runs over the (finitely many) anisotropic primes, $n_{1}, \ldots, n_{r}$ are the finitely many "sporadic" natural numbers not represented by $Q$, and $t_{1} \mathbb{Z}^{2}, \ldots, t_{m} \mathbb{Z}^{2}$ are finitely many spinor exceptional square classes which may not be represented. Thus, $E(f)$ is a subset of a union of finitely many square classes, and hence its asymptotic density is zero.

We then see that the local conditions at any odd prime $p$ are equivalent to those given in the theorem by Lemma 3.1 for $Q$. We will use the original form $f$ to investigate the local condition at $p=2$. A quick check shows that $T_{z}$ represents every integer modulo 8, and Hensel's lemma then shows that $T_{z}$ represents every integer 2-adically. Therefore, if $c$ is odd, then $c T_{z}$ represents every integer 2-adically. If $v_{2}(c)=1$, then $c T_{z}$ represents every even integer. Since $\operatorname{gcd}(a, b, c)=1$, either $a$ or $b$ is odd, hence every integer is 2-adically represented. If $v_{2}(c)=2$, then $c T_{z}$ 
represents every integer congruent to $4 \bmod 8$. Hence, we must represent 1,2 , and 3 modulo 4 with $a x^{2}+b y^{2}$. Without loss of generality, we assume that $b$ is odd. Then, $b$ is congruent to 1 or 3 modulo 4 , and so is $b y^{2}$ whenever $y$ is odd. If $a$ is odd, then either $a \equiv b(\bmod 4)$, in which case $-b(\bmod 4)$ is not represented, or $a \equiv-b(\bmod 4)$, in which case $2(\bmod 4)$ is not represented. Therefore, one sees $a \equiv 2(\bmod 4)$, which is equivalent to $v_{2}(a)=1$. Finally, if $v_{2}(c) \geq 3$, then $a x^{2}+b y^{2}$ cannot represent every integer modulo 8 because an odd square is always congruent to $1 \bmod 8$, so the local conditions are not satisfied.

Proof of Theorem 1.2. In this case the number of solutions to $n=f(x, y, z)=$ $a x^{2}+b T_{y}+c T_{z}$ equals the number of representations of $8 n+b+c$ by

$$
Q(x, y, z)=8 a x^{2}+b y^{2}+c z^{2}
$$

with $y$ and $z$ odd. Thus, we again only need to show that every integer is locally represented. The conditions given in the theorem for the odd primes are precisely those given by Lemma 3.1 For $p=2$ we again use the fact that $T_{y}$ and $T_{z}$ represent every integer 2-adically. Note that if $v_{2}(b) \leq 1$ or $v_{2}(c) \leq 1$, then every 2 -adic integer is represented because at least one of $a, b, c$ must be odd. Also, if $2 \leq v_{2}(c) \leq v_{2}(b)$, then not every integer is represented modulo 4 .

Proof of Theorem 1.3. Clearly, $f(x, y, z)=a T_{x}+b T_{y}+c T_{z}$ represents the integer $n$ if and only if

$$
Q(x, y, z)=a x^{2}+b y^{2}+c z^{2}
$$

represents $8 n+a+b+c$ with $x, y, z$ all odd. Again the local conditions at the odd primes are given by Lemma 3.1. For the 2-adic conditions, we simply note that one of $a, b, c$ is odd, so every 2 -adic integer is represented.

Proof of Corollary 1.4. We first note that if $b R a$, then $\left(\frac{b}{a}\right)=1$. Thus, if the conditions given in Theorems 1.1, 1.2, or 1.3 hold, then (by the multiplicative property of Jacobi symbols) we have

$$
1=\left(\frac{-b^{\prime} c^{\prime}}{a^{\prime}}\right)\left(\frac{-a^{\prime} c^{\prime}}{b^{\prime}}\right)\left(\frac{-a^{\prime} b^{\prime}}{c^{\prime}}\right)\left(\frac{2^{r}}{b^{\prime} c^{\prime}}\right)\left(\frac{2^{s}}{a^{\prime} b^{\prime}}\right)\left(\frac{2^{t}}{a^{\prime} c^{\prime}}\right),
$$

where $r, s, t$ are certain natural numbers.

By the law of quadratic reciprocity for Jacobi symbols,

$$
\begin{aligned}
& \left(\frac{-b^{\prime} c^{\prime}}{a^{\prime}}\right)\left(\frac{-a^{\prime} c^{\prime}}{b^{\prime}}\right)\left(\frac{-a^{\prime} b^{\prime}}{c^{\prime}}\right) \\
= & \left(\frac{-1}{a^{\prime} b^{\prime} c^{\prime}}\right) \cdot\left(\frac{b^{\prime}}{a^{\prime}}\right)\left(\frac{a^{\prime}}{b^{\prime}}\right) \cdot\left(\frac{c^{\prime}}{a^{\prime}}\right)\left(\frac{a^{\prime}}{c^{\prime}}\right) \cdot\left(\frac{c^{\prime}}{b^{\prime}}\right)\left(\frac{b^{\prime}}{c^{\prime}}\right) \\
= & (-1)^{\frac{a^{\prime}-1}{2}+\frac{b^{\prime}-1}{2}+\frac{c^{\prime}-1}{2}}(-1)^{\frac{a^{\prime}-1}{2} \cdot \frac{b^{\prime}-1}{2}+\frac{a^{\prime}-1}{2} \cdot \frac{c^{\prime}-1}{2}+\frac{b^{\prime}-1}{2} \cdot \frac{c^{\prime}-1}{2}} \\
= & (-1)^{\frac{a^{\prime}+1}{2} \cdot \frac{b^{\prime}+1}{2} \cdot \frac{c^{\prime}+1}{2}-\frac{a^{\prime}-1}{2} \cdot \frac{b^{\prime}-1}{2} \cdot \frac{c^{\prime}-1}{2}-1 .}
\end{aligned}
$$

Observe that $\frac{a^{\prime}+1}{2} \cdot \frac{b^{\prime}+1}{2} \cdot \frac{c^{\prime}+1}{2}$ and $\frac{a^{\prime}-1}{2} \cdot \frac{b^{\prime}-1}{2} \cdot \frac{c^{\prime}-1}{2}$ have opposite parity if and only if $a^{\prime} \equiv b^{\prime} \equiv c^{\prime}(\bmod 4)$. So the product of three Jacobi symbols is 1 if and only if $a^{\prime} \equiv b^{\prime} \equiv c^{\prime}(\bmod 4)$.

We finally deal with the 2 -power part. If $a^{\prime} \equiv b^{\prime} \equiv-c^{\prime}(\bmod 8)$, then

$$
1=\left(\frac{2}{a^{\prime} b^{\prime}}\right)=\left(\frac{2}{b^{\prime} c^{\prime}}\right)=\left(\frac{2}{a^{\prime} c^{\prime}}\right)
$$


which concludes the first statement. We now note that if $\pm a^{\prime} \equiv c^{\prime}+4(\bmod 8)$, then $\left(\frac{2}{a^{\prime}}\right)=-\left(\frac{2}{c^{\prime}}\right)$. Therefore, in the cases $a^{\prime} \equiv b^{\prime} \equiv c^{\prime}+4(\bmod 8)$ or $\pm a^{\prime} \equiv$ $-b^{\prime} \equiv c^{\prime}+4(\bmod 8)$ the Jacobi symbol from the 2-power part is $(-1)^{r+t}$, where $r$ and $t$ are as in equation (3.3). For (1), (4), and (5), we have $r=v_{2}(a)+1$, while $r=v_{2}(a)$ in the cases (2) and (3). For (1), we have $t=v_{2}(b)+1$, and otherwise $t=v_{2}(b)$. Thus for (1)-(3) we have $(-1)^{r+t}=(-1)^{v_{2}(a)+v_{2}(b)}$ and for (4)-(5) we have $(-1)^{r+t}=-(-1)^{v_{2}(a)+v_{2}(b)}$, from which we conclude the remaining two statements.

Proof of Corollary 1.5, By Theorem 1.1] if $a x^{2}+b y^{2}+2 c T_{z}$ or $a x^{2}+c y^{2}+2 b T_{z}$ is asymptotically universal, then we have (3.1). Similarly, if $a x^{2}+2 c y^{2}+b T_{z}$ or $a x^{2}+2 b y^{2}+c T_{z}$ is asymptotically universal, then we have

$$
-b c R a^{\prime},-2 a c R b^{\prime},-2 a b R c^{\prime} \text {. }
$$

Now assume that both (3.1) and (3.4) hold. We want to deduce a contradiction. (3.1) and (3.4) imply that $2 R b^{\prime}$ and $2 R c^{\prime}$. Recall that $v_{2}(b) \equiv v_{2}(c)(\bmod 2)$. So we have

$$
-b^{\prime} c^{\prime} R a^{\prime},-a^{\prime} c^{\prime} R b^{\prime},-a^{\prime} b^{\prime} R c^{\prime} .
$$

It follows that

$$
\left(\frac{-b^{\prime} c^{\prime}}{a^{\prime}}\right)=\left(\frac{-a^{\prime} c^{\prime}}{b^{\prime}}\right)=\left(\frac{-a^{\prime} b^{\prime}}{c^{\prime}}\right)=1
$$

Since $a^{\prime} \equiv b^{\prime} \equiv c^{\prime}(\bmod 4)$ fails, as in the proof of Corollary 1.4 we have

$$
\left(\frac{-b^{\prime} c^{\prime}}{a^{\prime}}\right)\left(\frac{-a^{\prime} c^{\prime}}{b^{\prime}}\right)\left(\frac{-a^{\prime} b^{\prime}}{c^{\prime}}\right)=-1 \text {. }
$$

So a contradiction occurs.

The following lemma gives a sufficient condition for a form not to be asymptotically universal. It will be helpful for our proofs in the next section.

Lemma 3.2. Let $a, b, c \in \mathbb{Z}^{+}$. For

$$
f(x, y, z)=a x^{2}+b y^{2}+c T_{z}, a x^{2}+b T_{y}+c T_{z}, a T_{x}+b T_{y}+c T_{z}
$$

we define $v_{f}=v_{2}(c), v_{2}(b+c), v_{2}(a+b+c)$, respectively. If $v_{f} \geq 3$, then $f$ is not asymptotically universal.

Proof. Assume that $v_{f} \geq 3$ and $f$ is asymptotically universal. We want to deduce a contradiction.

In the case $f=a x^{2}+b y^{2}+c T_{z}$, by Theorem 1.1 we have $8 \nmid c$, which contradicts $v_{f} \geq 3$.

Now suppose that $f=a x^{2}+b T_{y}+c T_{z}$. Since $4 \nmid b$ or $4 \nmid c$ by Theorem 1.2 , (up to symmetry) the vector $(b, c)$ modulo 8 is one of $(2,6),(5,3)$, or $(1,7)$. In the first case $a$ must be odd, while in the remaining two cases we have $b c \equiv 7$ $(\bmod 8)$ and hence $\left(\frac{2}{b c}\right)=1$. Therefore, Theorem 1.2 and equation (3.3) imply that $\left(\frac{-a^{\prime} b^{\prime}}{c^{\prime}}\right)\left(\frac{-a^{\prime} c^{\prime}}{b^{\prime}}\right)\left(\frac{-b^{\prime} c^{\prime}}{a^{\prime}}\right)=1$. However, the calculation from Corollary 1.4 shows that

$$
\left(\frac{-a^{\prime} b^{\prime}}{c^{\prime}}\right)\left(\frac{-a^{\prime} c^{\prime}}{b^{\prime}}\right)\left(\frac{-b^{\prime} c^{\prime}}{a^{\prime}}\right)=1
$$

if and only if $a^{\prime} \equiv b^{\prime} \equiv c^{\prime}(\bmod 4)$. Since $b^{\prime} \equiv 1(\bmod 4)$ and $c^{\prime} \equiv 3(\bmod 4)$, we are led to a contradiction. 
Finally we handle the case $f=a T_{x}+b T_{y}+c T_{z}$. By Theorem 1.3 and $v_{f} \geq 3$, the vector $(a, b, c)$ modulo 8 is one of $(8,1,7),(8,3,5),(2,5,1),(2,3,3),(2,7,7),(6,1,1)$, $(6,5,5),(6,3,7),(4,1,3),(4,5,7)$. The cases $(8,1,7)$ and $(8,3,5)$ are covered above. For the cases $(2,3,3),(2,7,7),(4,1,3),(4,5,7),(6,1,1),(6,5,5)$ we have

$$
\left(\frac{-a^{\prime} b^{\prime}}{c^{\prime}}\right)\left(\frac{-a^{\prime} c^{\prime}}{b^{\prime}}\right)\left(\frac{-b^{\prime} c^{\prime}}{a^{\prime}}\right)=-1 \text { and }\left(\frac{2^{v_{2}(a)}}{b^{\prime} c^{\prime}}\right)=1 \text {, }
$$

while in the cases $(2,5,1)$ and $(6,3,7)$ we have

$$
\left(\frac{-a^{\prime} b^{\prime}}{c^{\prime}}\right)\left(\frac{-a^{\prime} c^{\prime}}{b^{\prime}}\right)\left(\frac{-b^{\prime} c^{\prime}}{a^{\prime}}\right)=1 \text { and }\left(\frac{2}{b^{\prime} c^{\prime}}\right)=-1
$$

In view of (3.3), we get a contradiction.

\section{On ALmost universal FORMS}

In this section we investigate almost universal forms. We will determine when asymptotically universal forms are not almost universal. We first consider sums with two squares.

Proof of Theorem 1.6. Assume the conditions of Theorem 1.1. Recall that $n$ is represented by $f(x, y, z)=a x^{2}+b y^{2}+c T_{z}$ if and only $8 n+c$ is represented by

$$
Q(x, y, z)=8 a x^{2}+8 b y^{2}+c z^{2}
$$

with $z$ odd. Since $v_{2}(c) \leq 2$ there are no representations of $8 n+c$ by $Q(x, y, 2 z)$ due to congruence conditions modulo 8; thus the odd condition can be removed. Therefore,

$$
E(f)=\left\{\frac{n-c}{8}: n \equiv c(\bmod 8), Q(x, y, z)=n \text { has no integral solution }\right\}
$$

Let $t \mathbb{Z}^{2}$ be a spinor exceptional square class for the genus of $Q$ such that $t$ is squarefree and $t x^{2} \equiv c(\bmod 8)$ for some integer $x$. We will see below that $K=$ $\mathbb{Q}(\sqrt{-t a b c})$ will always be $\mathbb{Q}(i)$ or $\mathbb{Q}(\sqrt{-2})$. Thus by the results of Earnest, Hsia, and Hung $[8]$ is a spinor exception for the genus because $t x^{2}$ satisfying the SchulzePillot conditions will imply that $t$ satisfies the Schulze-Pillot conditions.

When $t$ is not represented by the spinor genus of $Q$, Schulze-Pillot 24] showed that for every prime $p$ splitting in $K$ we have that $t p^{2}$ is not represented by the spinor genus of $Q$, and hence not by $Q$ (see [25] for a full list of such properties). If $t$ is represented by the spinor genus of $Q$, then for each prime $p$ inert in $K$ we have that $t p^{2}$ is not primitively represented by the spinor genus of $Q$ [25]. Here a primitive representation means that $\operatorname{gcd}(x, y, z)=1$. Thus, for any squarefree $t$ represented by the spinor genus of $Q$ but not represented by $Q, t p^{2}$ is not represented when $p$ is inert in $K$, as the number of representations of $t p^{2}$ equals the number of (primitive) representations of $t$ plus the number of primitive representations of $t p^{2}$, and both of these are zero. Hence, in either case we have seen that there are infinitely many integers in $t \mathbb{Z}^{2}$ not represented by $Q$ so that $E(f)$ is infinite if such a $t$ exists. 
Next we show that if no such $t$ exists, then $E(f)$ is finite. By equations (3.2) and (4.1), if there is no such $t$ with $t x^{2} \equiv c(\bmod 8)$ for some $x \in \mathbb{Z}$, then

$$
\{8 n+c: n \in E(f)\} \subseteq\left(\bigcup_{j=1}^{r} \bigcup_{p}\left\{n_{j} p^{2 s}: s \in \mathbb{N}\right\}\right),
$$

where $n_{1}, \ldots, n_{r}$ are "sporadic" exceptions. Note that if every integer is represented $p$-adically by the quadratic form $8 a x^{2}+8 b y^{2}+c z^{2}$, then $p$ is not anisotropic. Assume $p \mid c$ and fix an integer $n$. Clearly, any $p$-adic solution to $Q(x, y, z)=n$ gives a solution to $Q(p x, p y, p z)=n p^{2}$. Since $Q$ satisfies the condition of Lemma 3.1 and for any fixed $y \in \mathbb{Z}$ relatively prime to $p$ the equation $a x^{2}=n p^{2}-b y^{2}$ has a solution with $x$ relatively prime to $p$, there are more solutions to the equation $Q(x, y, z)=n p^{2}$ than to the equation $Q(x, y, z)=n$; hence $p$ is not anisotropic.

Thus, the only possible anisotropic prime is $p=2$, and hence

$$
\{8 n+c: n \in E(f)\} \subseteq \bigcup_{j=1}^{r}\left\{n_{j} 2^{2 s}: s \in \mathbb{N}\right\} .
$$

As $v_{2}(c) \leq 2$, we have

$$
\{8 n+c: n \in E(f)\} \subseteq \bigcup_{j=1}^{r}\left\{n_{j} 2^{2 s}: s \in\{0,1,2\}\right\},
$$

which shows that $E(f)$ is finite.

We now use Schulze-Pillot's classification 24 to determine the spinor exceptional square classes $t_{i} \mathbb{Z}^{2}$. Let a spinor exceptional square class $t \mathbb{Z}^{2}$ be given. Earnest, Hsia, and Hung showed that if an odd prime $p$ is ramified in $K=\mathbb{Q}(\sqrt{-t d})$, then $Q_{p} \cong\left\langle u_{1}, u_{2} p^{r}, u_{3} p^{s}\right\rangle$ with $u_{i}$ units in $\mathbb{Z}_{p}$ and $0<r<s$ (cf. [8, Theorem 1(b)]). However, since $p$ divides at most one of $a, b, c$, this cannot occur. It follows that $p$ is unramified in $K$, hence $K=K_{a b c}$ or $K=K_{2 a b c}$.

Recall that $\mathcal{S F}\left(a^{\prime} b^{\prime} c^{\prime}\right)$ is the odd squarefree part of $a b c$. Assume that a prime $p$ dividing $\mathcal{S F}\left(a^{\prime} b^{\prime} c^{\prime}\right)$ is not split in $K$. Then, by Theorem 1(a) of Earnest, Hsia, and Hung [8], we have $Q_{p} \cong\left\langle u_{1}, u_{2} p^{2 r}, u_{3} p^{2 s}\right\rangle$ from the necessary condition given by Kneser [14. But this would contradict the fact that $v_{p}(a b c)$ is odd. Conversely, when $p$ is split in $K$, Earnest, Hsia and Hung showed that the local conditions for $t$ to be a spinor exception are satisfied (cf. 8]). If $p$ is odd and $v_{p}(a b c)$ is even, then $[\underline{8}$, Theorem $1(\mathrm{a})]$ shows that $t \not \equiv 0(\bmod p)$ satisfies the necessary and sufficient conditions. Thus, the only possible spinor exceptional square classes are given by $t=\mathcal{S F}\left(a^{\prime} b^{\prime} c^{\prime}\right)$ or $t=2 \mathcal{S F}\left(a^{\prime} b^{\prime} c^{\prime}\right)$. If $t \not \equiv 2^{-2 s} c(\bmod 8)$ for some $s \in \mathbb{N}$ with $2 s \leq v_{2}(c)$, then this spinor exceptional square class will not occur in our consideration. Hence we conclude that $t=\mathcal{S F}\left(a^{\prime} b^{\prime} c\right)$. Since tabc times a suitable square equals $a a^{\prime} b b^{\prime} c^{2}$, we have $K=K_{a b c^{\prime}}$.

From the above we see that every $p \mid \mathcal{S F}\left(a^{\prime} b^{\prime} c^{\prime}\right)$ must be split in $K$, which gives condition (2). If $Q$ represents $t$, then $Q$ also represents $t \mathbb{Z}^{2}$ (not necessarily primitively), and hence condition (3) is necessary.

We finally deal with the 2 -adic conditions. Let $\beta$ be a prime ideal of $O_{K}$ dividing 2. Since $K=\mathbb{Q}(i)$ or $K=\mathbb{Q}(\sqrt{-2})$, the 2 -adic completion $K_{\beta} / \mathbb{Q}_{2}$ is ramified. After division by common powers of 2 , we get

$$
Q_{2} \cong\left\langle c^{\prime}, 2^{r} b^{\prime}, 2^{s} a^{\prime}\right\rangle
$$

where $3-v_{2}(c)+v_{2}(b)=r \leq s=3-v_{2}(c)+v_{2}(a)$. 
We now separate into cases depending on $v_{2}(c)$. First we consider the case where $v_{2}(c)=2$. In this case, we divide the equation $Q(x, y, z)=8 n+c$ by 4 to find that representation of $n$ by $f$ is equivalent to representation of $2 n+1$ by $Q^{\prime}(x, y, z)=2 a x^{2}+2 b y^{2}+c^{\prime} z^{2}$. We recall by the conditions of Theorem 1.1 that $v_{2}(a)=1$ and $v_{2}(b)=0$. Thus, $L_{2} \cong\left\langle c^{\prime}, 2 b^{\prime}, 4 a^{\prime}\right\rangle$. Since $r \leq 3$ and $s \leq 2$, the conditions of Theorem 2.2 are always satisfied for $v_{2}(4 t)=2$. Therefore every sufficiently large integer is always represented in this case.

For the remaining case $\mathcal{S F}\left(a^{\prime} b^{\prime} c\right)=t \equiv c(\bmod 8)$, we conclude that $a^{\prime} b^{\prime} \equiv 1$ $\left(\bmod 2^{3-v_{2}(c)}\right)$. Hence $a^{\prime} \equiv b^{\prime}\left(\bmod 2^{3-v_{2}(c)}\right)$, which gives the first assertion of condition (11). Assume first that $c$ is odd. When $r \geq 5$, Earnest and Hsia [7] showed that

$$
\theta\left(O^{+}\left(\langle a, b, c\rangle_{2}\right)\right)=\theta\left(O^{+}\left(\langle a c, b c\rangle_{2}\right)\right)
$$

Since $a^{\prime} \equiv b^{\prime}(\bmod 8)$ and scaling does not affect the spinor norm, the lattice on the right-hand side is equivalent to $\left\langle 1,2^{s-r}\right\rangle_{2}$. If $s=r$, then this is precisely $N_{2}(\mathbb{Q}(i))$, as desired. Checking each case of Theorem 2.1 shows that $\theta\left(O^{+}\left(\left\langle 1,2^{s-r}\right\rangle_{2}\right)\right) \subseteq$ $N_{2}(K)$, since $K=\mathbb{Q}(i)$ if $s-r$ is even, and $K=\mathbb{Q}(\sqrt{-2})$ if $s-r$ is odd. Theorem 2.2 indicates that when $r \geq 5$, the sufficient conditions are also satisfied. Hence, if $4 \mid b$, then $t$ is a spinor exception. For $2 \| b$ we have $5 \in \theta\left(O^{+}\left(\left\langle c, 2^{r} b^{\prime}\right\rangle_{2}\right)\right) \notin N_{2}(\mathbb{Q}(\sqrt{-2}))$; it follows that $K=\mathbb{Q}(i)$ and hence $s$ is even. But, when $r$ and $s$ have the same parity, none of the conditions of Theorem 2.2(1) is satisfied when $r \geq 4$; therefore $t$ is a spinor exception. For $r=3$, Theorem 2.1 implies that $K=\mathbb{Q}(\sqrt{-2})$ and $b \equiv c$ $(\bmod 8)$, and hence $s$ is even. If $s=4$, then Theorem 2.2 of Earnest and Hsia [7] shows that $\theta\left(O^{+}\left(\langle a, b, c\rangle_{2}\right)\right)=\mathbb{Q}_{2}^{\times}$. Therefore, $v_{2}(a) \geq 3$ is odd and $8 \mid(b-c)$ in this case. But then Theorem 2.2(2)(c) is not satisfied since $s>2$, so it follows that $t$ is a spinor exception.

In the case $2 \mid c$, we have $2 \nmid b$. Thus we get $\left\langle c^{\prime}, 4 b^{\prime}, 2^{s} a^{\prime}\right\rangle_{2}$ after division by 2 . In view of the sublattice $\left\langle c^{\prime}, 4 b^{\prime}\right\rangle_{2}$, we have $K=\mathbb{Q}(i)$, and hence $2 \mid s$. If $s=2$, then taking the product of symmetries $\sigma=\tau_{2 x_{1}+x_{2}+x_{3}} \tau_{x_{1}}$ gives $\theta(\sigma)=4\left(c^{\prime}+b^{\prime}+a^{\prime}\right) c^{\prime} \notin$ $N_{2}(\mathbb{Q}(i))$ because each of $a^{\prime}, b^{\prime}, c^{\prime}$ must be congruent to $1 \bmod 4$ by condition (2). Therefore $v_{2}(a)>0$ is even so that $2 \mid a$ and $v_{2}(a) \equiv c(\bmod 2)$. In this case $L^{\prime}=\left\langle c^{\prime}, 4 b^{\prime}, 2^{s} a^{\prime}\right\rangle_{2}$ where $L^{\prime}$ is as defined in Theorem 2.2(1), so $\theta\left(O^{+}\left(L^{\prime}\right) \subseteq N_{2}(K)\right.$. None of the conditions in Theorem $2.2(1)(\mathrm{c})-(\mathrm{d})$ can be satisfied, so $t$ is a spinor exception.

Proof of Corollary 1.7. For any $n \in \mathbb{N}$ we have

$$
\begin{aligned}
& 2 n+1=2 a x^{2}+2 b y^{2}+c z^{2} \text { for some } x, y, z \in \mathbb{Z} \\
\Longleftrightarrow & 2 n+1=2 a x^{2}+2 b y^{2}+c(2 z+1)^{2} \text { for some } x, y, z \in \mathbb{Z} \\
\Longleftrightarrow & n-\frac{c-1}{2}=a x^{2}+b y^{2}+4 c T_{z} \text { for some } x, y, z \in \mathbb{Z} .
\end{aligned}
$$

By Theorem 1.6 and Theorem 1.1

$$
\begin{aligned}
& a x^{2}+b y^{2}+4 c T_{z} \text { is almost universal } \\
\Longleftrightarrow & a x^{2}+b y^{2}+4 c T_{z} \text { is asymptotically universal } \\
\Longleftrightarrow & 2 \| a b,-8 b c R a^{\prime},-8 a c R b^{\prime} \text {, and }-a b R c .
\end{aligned}
$$

So the first part of Corollary 1.7 follows. 
When $n \in \mathbb{N}$, clearly

$$
\begin{aligned}
2 n+1 & =2 a x^{2}+c\left(y^{2}+z^{2}\right) \text { for some } x, y, z \in \mathbb{Z} \\
\Longleftrightarrow 2 n+1 & =2 a x^{2}+4 c y^{2}+c z^{2} \text { for some } x, y, z \in \mathbb{Z} .
\end{aligned}
$$

In the case $b=2 c$,

$$
\begin{aligned}
& 2 \| a b,-2 b c R a^{\prime},-2 a c R b^{\prime}, \text { and }-a b R c \\
\Longleftrightarrow & c=1,2 \nmid a, \text { and }-1 R a^{\prime} .
\end{aligned}
$$

So we also have the second part of Corollary 1.7.

Proof of Corollary 1.8. In light of Theorem 1.1 .

$$
\begin{aligned}
& a x^{2}+b y^{2}+2 T_{z} \text { is asymptotically universal } \\
\Longleftrightarrow & -b R a^{\prime} \text { and }-a R b \\
\Longleftrightarrow & a x^{2}+y^{2}+2 b T_{z} \text { is asymptotically universal }
\end{aligned}
$$

and

$$
\begin{aligned}
& a x^{2}+2 y^{2}+b T_{z} \text { is asymptotically universal } \\
\Longleftrightarrow & -b R a^{\prime} \text { and }-2 a R b \\
\Longleftrightarrow & a x^{2}+2 b y^{2}+T_{z} \text { is asymptotically universal }
\end{aligned}
$$

Now assume that $-a R b$ and $-b R a^{\prime}$. Then both $a x^{2}+b y^{2}+2 T_{z}$ and $a x^{2}+y^{2}+$ $2 b T_{z}$ are asymptotically universal. Recall that $\mathcal{S} \mathcal{F}\left(a^{\prime} b\right)=\mathcal{S} \mathcal{F}\left(a^{\prime}\right) \mathcal{S F}(b)$ has a prime divisor $p \equiv 3(\bmod 4)$. Whether $v_{2}(a)$ is even or odd, we cannot have both (1) and (2) of Theorem 1.6 for either of the two forms. It follows that $a x^{2}+b y^{2}+2 T_{z}$ and $a x^{2}+y^{2}+2 b T_{z}$ are almost universal.

Suppose that $-2 a R b$ and $-b R a^{\prime}$. Then both $a x^{2}+2 y^{2}+b T_{z}$ and $a x^{2}+2 b y^{2}+T_{z}$ are asymptotically universal. As $2 b \equiv 2 \not \equiv 0(\bmod 4)$ and not all prime divisors of $\mathcal{S F}\left(a^{\prime} b\right)$ are congruent to $3 \bmod 4$, we cannot have both (1) and (2) of Theorem 1.6 for either of the two forms. So $a x^{2}+2 y^{2}+b T_{z}$ and $a x^{2}+2 b y^{2}+T_{z}$ must be almost universal. We are done.

Proof of Corollary 1.9, (i) By Theorem 1.1. $a x^{2}+y^{2}+T_{z}$ is asymptotically universal $\Longleftrightarrow-2 R a^{\prime}$, i.e., $\left(\frac{-2}{p}\right)=1$ for each prime divisor $p$ of $a^{\prime}$ $\Longleftrightarrow$ all odd prime divisors of $a$ are congruent to 1 or $3 \bmod 8$.

Similarly,

$$
a x^{2}+2 y^{2}+2 T_{z} \text { is asymptotically universal }
$$

$\Longleftrightarrow$ all prime divisors of $a$ are congruent to 1 or $3 \bmod 8$.

Now suppose that $-2 R a^{\prime}$. As each prime $p \equiv 1,3(\bmod 8)$ can be written in the form $x^{2}+2 y^{2}$ with $x, y \in \mathbb{Z}$, and

$$
\left(x_{1}+2 y_{1}^{2}\right)\left(x_{2}^{2}+2 y_{2}^{2}\right)=\left(x_{1} x_{2}-2 y_{1} y_{2}\right)^{2}+2\left(x_{1} y_{2}+x_{2} y_{1}\right)^{2},
$$

we can write $\mathcal{S F}\left(a^{\prime}\right)$ in the form $x_{0}^{2}+2 y_{0}^{2}$ with $x_{0}, y_{0} \in \mathbb{Z}$ since all prime divisors of $a^{\prime}$ are congruent to 1 or 3 modulo 8 . If $a^{\prime} \equiv 1(\bmod 8)$, then $\mathcal{S F}\left(a^{\prime}\right) \equiv 1(\bmod 8)$ and hence $y_{0}$ must be even, so the equation $8\left(a x^{2}+y^{2}\right)+z^{2}=\mathcal{S F}\left(a^{\prime}\right)$ has a 
solution $(x, y, z)=\left(0, y_{0} / 2, x_{0}\right)$, which violates $(3)$ in Theorem 1.6 with $b=c=1$. If $a^{\prime} \equiv 3(\bmod 8)$, then we don't have $(1)$ in Theorem 1.6 with $b=c=1$. Therefore, by Theorem 1.6, $a x^{2}+y^{2}+T_{z}$ must be almost universal. When $a$ is odd, we have $4 \nmid a$ and $v_{2}(2) \not \equiv 2(\bmod 2)$; therefore $a x^{2}+2 y^{2}+2 T_{z}$ is almost universal by Theorem 1.6 with $c=2$.

(ii) By Theorem 1.1,

$$
\begin{aligned}
& a x^{2}+2 y^{2}+T_{z}\left(\text { or } a x^{2}+y^{2}+2 T_{z}\right) \text { is asymptotically universal } \\
\Longleftrightarrow & -1 R a^{\prime} \text {, i.e., }\left(\frac{-1}{p}\right)=1 \text { for each prime divisor } p \text { of } a^{\prime} \\
\Longleftrightarrow & \text { all odd prime divisors of } a \text { are congruent to } 1 \bmod 4 .
\end{aligned}
$$

Similarly,

$$
a x^{2}+4 y^{2}+2 T_{z}\left(\text { or } a x^{2}+2 y^{2}+4 T_{z}\right) \text { is asymptotically universal }
$$

$\Longleftrightarrow$ all prime divisors of $a$ are congruent to $1 \bmod 4$.

Below we assume that $-1 R a^{\prime}$. It is well known that each prime $p \equiv 1(\bmod 4)$ is a sum of two squares (of integers) and

$$
\left(x_{1}+y_{1}^{2}\right)\left(x_{2}^{2}+y_{2}^{2}\right)=\left(x_{1} x_{2}-y_{1} y_{2}\right)^{2}+\left(x_{1} y_{2}+x_{2} y_{1}\right)^{2} .
$$

So we can write $\mathcal{S F}\left(a^{\prime}\right)$ in the form $x_{0}^{2}+y_{0}^{2}$ with $x_{0}$ odd and $y_{0}$ even (since all prime divisors of $a^{\prime}$ are congruent to $\left.1 \bmod 4\right)$. Thus the equation $4\left(a x^{2}+y^{2}\right)+z^{2}=\mathcal{S F}\left(a^{\prime}\right)$ has a solution $(x, y, z)=\left(0, y_{0} / 2, x_{0}\right)$, which violates $(3)$ in Theorem 1.6 with $b=1$ and $c=2$. So $a x^{2}+y^{2}+2 T_{z}$ is almost universal. If $a^{\prime} \equiv 1(\bmod 8)$, then $\mathcal{S} \mathcal{F}\left(a^{\prime}\right) \equiv$ $a^{\prime} \not \equiv 5(\bmod 8)$ and hence $4 \mid y_{0}$, so the equation $8\left(a x^{2}+2 y^{2}\right)+z^{2}=\mathcal{S F}\left(a^{\prime}\right)$ has an integral solution $(x, y, z)=\left(0, y_{0} / 4, x_{0}\right)$, which violates $(3)$ in Theorem 1.6 for the form $a x^{2}+2 y^{2}+T_{z}$. If $a^{\prime} \not \equiv 1(\bmod 8)$, then we don't have $(1)$ in Theorem 1.6 for the form $a x^{2}+2 y^{2}+T_{z}$. Thus, in view of Theorem 1.6. $a x^{2}+2 y^{2}+T_{z}$ is also almost universal.

Now we also assume that $a$ is odd. Note that the equation $2\left(a x^{2}+2 y^{2}\right)+$ $z^{2}=\mathcal{S F}\left(a^{\prime}\right)$ has an integral solution $(x, y, z)=\left(0, y_{0} / 2, x_{0}\right)$. Also, $v_{2}(4) \equiv$ $v_{2}(a)(\bmod 2)$,

$$
a \equiv \mathcal{S F}(a) \equiv 1(\bmod 8) \Longrightarrow 4\left(a 0^{2}+4 y^{2}\right)+z^{2}=\mathcal{S F}(a) \text { for some } y, z \in \mathbb{Z},
$$

and

$$
a \equiv \mathcal{S F}(a) \equiv 5(\bmod 8) \Longrightarrow 4\left(a x^{2}+4 y^{2}\right)+z^{2}=\mathcal{S F}(a) \text { for no } x, y, z \in \mathbb{Z} .
$$

Thus, by Theorem 1.6. the form $a x^{2}+2 y^{2}+4 T_{z}$ is almost universal, and $a x^{2}+$ $4 y^{2}+2 T_{z}$ is almost universal if and only if $a \equiv 1(\bmod 8)$.

The proof of is Corollary 1.9 is now complete.

Proof of Corollary 1.10, By Theorem 1.1 $a x^{2}+3 y^{2}+T_{z}$ (resp., $a x^{2}+y^{2}+3 T_{z}$, $\left.a x^{2}+2 y^{2}+6 T_{z}, a x^{2}+6 y^{2}+2 T_{z}\right)$ is asymptotically universal if and only if both $-6 R a^{\prime}$ and $a \equiv 1(\bmod 3)($ resp., $a \equiv 2(\bmod 3), a \equiv 1(\bmod 6), a \equiv 5(\bmod 6))$. Observe that $-6 R a^{\prime}$ if and only if for each odd prime divisor $p$ of $a$ we have

$$
\left(\frac{2}{p}\right)=\left(\frac{-3}{p}\right)=\left(\frac{p}{3}\right) \text {, i.e., } p \equiv 1,5,7,11(\bmod 24) \text {. }
$$

For odd positive integers $b$ and $c$ not satisfying $a^{\prime} \equiv b \equiv c(\bmod 8)$, by Theorem 1.6 the form $a x^{2}+b T_{y}+c T_{z}$ is almost universal if and only if it is asymptotically universal. Thus $a x^{2}+3 y^{2}+T_{z}$ (or $a x^{2}+y^{2}+3 T_{z}$ ) is almost universal if and only 
if it is asymptotically universal. If $a$ is odd, then $4 \nmid a$ and $v_{2}(2)=v_{2}(6)=1 \not \equiv 6 \equiv$ $2(\bmod 2)$; thus by Theorem 1.6 the form $a x^{2}+2 T_{y}+6 T_{z}\left(\right.$ or $\left.a x^{2}+6 y^{2}+2 T_{z}\right)$ is almost universal if and only if it is asymptotically universal.

Combining the above, we have completed the proof of Corollary 1.10 ,

Proof of Corollary 1.11, Let $k, l \in \mathbb{N}$ with $k \geq l$. By Theorem 1.1, the form $2^{k} x^{2}+$ $2^{l} y^{2}+m T_{z}$ is asymptotically universal if and only if $-2^{k+l} R m^{\prime}$ and

$$
4 \nmid m \text { or }(4 \| m \& k=1 \& l=0) \text {. }
$$

Assume that $2^{k} x^{2}+2^{l} y^{2}+m T_{z}$ is asymptotically universal. As $v_{2}(m)<3$ and $2 \nmid \mathcal{S F}\left(m^{\prime}\right)$, the equation

$$
2^{3-v_{2}(m)}\left(2^{k} x^{2}+2^{l} y^{2}\right)+m^{\prime} z^{2}=\mathcal{S F}\left(m^{\prime}\right)
$$

has no integral solution if and only if $m^{\prime}$ is not squarefree (i.e., $\left.\mathcal{S F}\left(m^{\prime}\right)<m^{\prime}\right)$. Thus, by Theorem 1.6, the form $2^{k} x^{2}+2^{l} y^{2}+m T_{z}$ is not almost universal if and only if $k>0$ and $4 \nmid m$ and

$$
\left\{\begin{array}{l}
l \leq 1 \Longrightarrow k \equiv m(\bmod 2), \\
l=0 \& 2 \nmid m \Longrightarrow k \geq 3 \& m \equiv 1(\bmod 8) .
\end{array}\right.
$$

In view of the above, we have the desired results in Corollary 1.11.

Proof of Corollary 1.12, By Theorem 1.1 .

$$
a x^{2}+6^{3} y^{2}+T_{z} \text { is asymptotically universal }
$$

$$
\begin{aligned}
& \Longleftrightarrow-2^{4} 3^{3} R a^{\prime} \text { and }-2 a R 3^{3} \\
& \Longleftrightarrow-3 R a^{\prime} \text { and }-2 a R 3 \\
& \Longleftrightarrow a \equiv 1(\bmod 3) \text { and }\left(\frac{p}{3}\right)=\left(\frac{-3}{p}\right)=1 \text { for each prime divisor } p \text { of } a^{\prime} \\
& \Longleftrightarrow \text { all prime divisors of } a^{\prime} \text { are congruent to } 1 \bmod 3, \text { and } 2 \mid v_{2}(a) .
\end{aligned}
$$

Also,

$$
\begin{aligned}
& a x^{2}+2 \cdot 5^{3} y^{2}+T_{z} \text { is asymptotically universal } \\
\Longleftrightarrow & -2^{2} 5^{3} R a^{\prime} \text { and }-2 a R 5^{3} \\
\Longleftrightarrow & -5 R a^{\prime} \text { and }-2 a R 5 \\
\Longleftrightarrow & a \equiv \pm 2(\bmod 5) \text { and }\left(\frac{-5}{p}\right)=1 \text { for each prime divisor } p \text { of } a^{\prime} .
\end{aligned}
$$

For an odd prime $p$, clearly

$$
\left(\frac{-5}{p}\right)=1 \Longleftrightarrow\left(\frac{-1}{p}\right)=\left(\frac{p}{5}\right) \Longleftrightarrow p \equiv 1,3,7,9(\bmod 20) \Longleftrightarrow 2 \mid\left\lfloor\frac{p}{10}\right\rfloor .
$$

(i) Under the supposition, $a x^{2}+216 y^{2}+T_{z}$ is asymptotically universal by the above. If $8\left(a x^{2}+216 y^{2}\right)+z^{2}=\mathcal{S F}\left(3^{3} a^{\prime}\right)=3 \mathcal{S F}\left(a^{\prime}\right)$ for some $x, y, z \in \mathbb{Z}$, then we must have $x=0$ (since $8 a>3 a$ ) and $3 \mid z$, which contradicts the fact that $3 \nmid \mathcal{S F}\left(a^{\prime}\right)$. So the equation $8\left(a x^{2}+216 y^{2}\right)+z^{2}=\mathcal{S F}\left(3^{3} a^{\prime}\right)$ has no integral solutions. Applying Theorem 1.6 we find that $a x^{2}+216 y^{2}+T_{z}$ is not almost universal if and only if $a^{\prime} \equiv 3^{3}(\bmod 8)$ and all prime divisors of $\mathcal{S F}\left(3^{3} a^{\prime}\right)=3 \mathcal{S F}\left(a^{\prime}\right)$ are congruent to 1 or $3 \bmod 8$. Since $\mathcal{S F}\left(a^{\prime}\right) \equiv a^{\prime}(\bmod 8)$ and each prime divisor of $a^{\prime}$ is congruent to $1 \bmod 3$, the desired result follows. 
(ii) Under the assumption, $a=2^{v_{2}(a)} a^{\prime} \equiv \pm 2(\bmod 5)$, and hence $a x^{2}+250 y^{2}+$ $T_{z}$ is asymptotically universal. Note that the equation $8\left(a x^{2}+250 y^{2}\right)+z^{2}=$ $\mathcal{S F}\left(5^{3} a^{\prime}\right)=5 \mathcal{S F}\left(a^{\prime}\right)$ has no integral solutions. In view of Theorem [1.6 $a x^{2}+$ $250 y^{2}+T_{z}$ is not almost universal if and only if $a^{\prime} \equiv 5^{3} \equiv 5(\bmod 8)$ and all prime divisors of $\mathcal{S F}\left(5^{3} a^{\prime}\right)=5 \mathcal{S F}\left(a^{\prime}\right)$ are congruent to $1 \bmod 4$. Since $a^{\prime} \equiv \pm 1(\bmod 10)$ and each prime divisor of $a^{\prime}$ is congruent to one of $1,3,7,9$ modulo 20, we finally obtain the desired result.

Proof of Theorem 1.13. As in Theorem 1.6 $f$ will not be almost universal only if there is a relevant anisotropic prime or a spinor exceptional square class with the correct congruence conditions modulo 8 for one of the quadratic forms occuring in the inclusion/exclusion of theta series

$$
\theta_{Q(x, y, z)}:=\theta_{Q^{\prime}(x, y, z)}-\theta_{Q^{\prime}(x, 2 y, z)}-\theta_{Q^{\prime}(x, y, 2 z)}+\theta_{Q^{\prime}(x, 2 y, 2 z)},
$$

where $Q^{\prime}(x, y, z)=8 a x^{2}+b y^{2}+c z^{2}$. We will first show that there are no relevant anisotropic primes. The conditions given by Theorem 1.2 imply that every odd prime $p$ is not anisotropic. By Lemma 3.2 the prime 2 is never relevant because the congruence condition implies that the 2-adic order is at most two.

Also as in Theorem 1.6. the local conditions at each odd prime imply that the only possible spinor exceptional square classes are $t \mathbb{Z}^{2}$ with $t=\mathcal{S F}\left(a^{\prime} b^{\prime} c^{\prime}\right)$ or $t=$ $2 \mathcal{S F}\left(a^{\prime} b^{\prime} c^{\prime}\right)$. Moreover, the sufficient local conditions for the odd primes are satisfied if and only if every prime divisor of $\mathcal{S F}\left(a^{\prime} b^{\prime} c^{\prime}\right)$ is split in $K=\mathbb{Q}(\sqrt{-2 a b c t})$.

If $t$ is a spinor exception for the genus of $Q^{\prime}(x, y, z)$, then $t$ is a spinor exception for the genus of $Q^{\prime}(x, 2 y, z)$ and condition (3) implies that $t$ is represented the same number of times by each quadratic form. If $t$ is not represented by the spinor genus of $Q^{\prime}$, then $t p^{2}$ is also not (primitively) represented, where $p$ is an odd prime split in $K$. If $t$ is represented by the spinor genus of $Q^{\prime}$, then $t p^{2}$ is not primitively represented, where $p$ is an inert prime. In either case $t p^{2}$ will also clearly not be primitively represented by $Q^{\prime}(x, 2 y, z)$, so $t p^{2}$ is not represented by $Q$. Also, if $t$ is not a spinor exception for $Q^{\prime}(x, y, z)$ or $Q^{\prime}(x, 2 y, z)$, then $E(f)$ is finite. Therefore, for $E(f)$ to be infinite, it is sufficient that $t$ is a spinor exception for the genus of $Q^{\prime}$, while it is necessary that $t$ is a spinor exception for the genus of $Q^{\prime}(x, 2 y, z)$.

We now break into cases depending on $v_{2}(b+c)$. Since $v_{2}(b+c)<3$ by Lemma 3.2 . we begin with the case $4 \| b+c$. Without loss of generality we assume $v_{2}(c) \leq v_{2}(b)$. Since $v_{2}(c)<2$ by local conditions, congruence conditions imply in this case that $v_{2}(b)=v_{2}(c) \leq 1$. If $v_{2}(c)=v_{2}(b)=1$, then $a$ is odd, and after division by common 2-powers we get $L=\left\langle c^{\prime}, b^{\prime}, 4 a^{\prime}\right\rangle_{2}$, so Theorem 2.2(1) is not satisfied because $v_{2}(4 t)=2 \geq s$. If $v_{2}(b)=v_{2}(c)=0$, then congruence conditions, without loss of generality, give $(b \equiv 1(\bmod 8) \& c \equiv 3(\bmod 8))$ or $(b \equiv 5(\bmod 8) \& c \equiv 7$ $(\bmod 8))$. In the second case not all prime divisors of $\mathcal{S F}\left(a^{\prime} b^{\prime} c^{\prime}\right)$ split in $K$. In the first case we must have $K=\mathbb{Q}(\sqrt{-2})$, and hence $s$ is odd. But then $v_{2}\left(2^{s} t\right)$ is odd and Theorem 2.2(2) implies that $0=v_{2}(b)>0$, thus $t$ is not a spinor exception.

For the remaining cases we note that $t \equiv b+c(\bmod 8)$, so we must have $v_{2}(t)=$ $v_{2}(b+c)$. Conditions (11), (2), and (3) now follow immediately. First consider $v_{2}(b) \geq 5$ odd. Then $\theta_{Q}=\theta_{Q^{\prime}(x, y, z)}-\theta_{Q^{\prime}(x, 2 y, z)}$, where $Q^{\prime}(x, y, z)=8 a x^{2}+b y^{2}+c z^{2}$. If $c$ is even, then we have $L_{2}=\left\langle c^{\prime}, 4 a^{\prime}, 2^{r-1} b^{\prime}\right\rangle_{2}$ and $K=\mathbb{Q}(i)$. In this case, Earnest and Hsia proved that the spinor norm can be considered only on $2 \times 2$ sublattices. Since $r-1$ is even, Theorem 2.1 shows that $\theta\left(O^{+}\left(L_{2}\right)\right) \subseteq N_{2}(K)$. Moreover, Theorem 2.2(1)(c)-(d) cannot be satisfied, so $t$ is a spinor exception. If $c$ is odd and 
$4 \mid a$, then $r \geq 5$, so the spinor norm again equals the spinor norm on 2-dimensional sublattices. Since $\mathcal{S} \mathcal{F}\left(a^{\prime} b^{\prime} c^{\prime}\right) \equiv(b+c)^{\prime} \equiv c^{\prime}(\bmod 8)$, we have $a^{\prime} \equiv b^{\prime}(\bmod 8)$. Therefore, Theorem 2.1 shows that the spinor norm on each sublattice gives a subset of $N_{2}(K)$. Since $r \geq 5$ none of the conditions of Theorem 2.2 is satisfied, and hence $t$ is a spinor exception. For $r=v_{2}(a)+3<5$, we have $\left\langle c^{\prime}, 2^{r} a^{\prime}, 2^{s} b^{\prime}\right\rangle_{2}$. Applying Theorem 2.1 to the the sublattice $\left\langle c^{\prime}, 2^{r} a^{\prime}\right\rangle_{2}$, we get $K=\mathbb{Q}\left(\sqrt{-2^{r}}\right)$, and it follows that $s$ must be even. Therefore $t$ is not a spinor exception for $Q^{\prime}(x, y, z)$ or $Q^{\prime}(x, 2 y, z)$.

For $v_{2}(b) \geq 6$ even and $c$ even, in view of the sublattice $\left\langle 2 c^{\prime}, 8 a\right\rangle_{2}$ and Theorem 2.1. we have $K=\mathbb{Q}(i)$, which implies that $v_{2}(b)$ must be odd. For $v_{2}(b) \geq 6$ even and $c$ odd, Earnest and Hsia showed that we may reduce the problem to 2 -dimensional sublattices. If $a$ is odd, then the sublattice $\langle c, 8 a\rangle_{2}$ gives the set $\left\{\gamma:(\gamma,-2 a c)_{2}=1\right\}$, which is a subgroup of $N_{2}(\mathbb{Q}(\sqrt{-2}))$ if and only if $a \equiv c$ $(\bmod 8)$. Theorem $2.2(2)(\mathrm{c})$ shows that $t$ is a spinor exception in this case, as $s>2$. When $a$ is even, we again note that $a^{\prime} \equiv b^{\prime}(\bmod 8)$ by condition (1) and that Theorem 2.1 implies that $\theta\left(O^{+}\left(\langle 8 a, b, c\rangle_{2}\right)\right) \subseteq N_{2}(K)$.

For $v_{2}(b)<3$, inclusion/exclusion gives $\theta_{Q}=\theta_{Q^{\prime}}$. For $b$ odd, the sublattice $\langle 1, b c\rangle_{2}$ gives the spinor norm $Q\left(x_{1}+2 x_{2}\right) Q\left(x_{1}\right) \mathbb{Q}_{2}^{\times^{2}}=5 \mathbb{Q}^{\times^{2}} \notin N_{2}(\mathbb{Q}(\sqrt{-2}))$, so $K=\mathbb{Q}(i)$ and $v_{2}(a)$ is even. We then note that condition (11) gives $1=\mathcal{S F}\left(a^{\prime} b^{\prime} c^{\prime}\right) \equiv$ $(b+c)^{\prime}(\bmod 4)$, so that $b \equiv c(\bmod 8)$. For $s=3+v_{2}(a) \geq 5$, the problem is now reduced to considering 2-dimensional sublattices, and we are done since $\theta\left(O^{+}\left(\langle 1,1\rangle_{2}\right)\right)=N_{2}(\mathbb{Q}(i))$ and $a^{\prime} \equiv 1(\bmod 4)$ by condition (2). In this case $L^{\prime \prime}=L_{2}$ and $\theta\left(O^{+}\left(L^{\prime}\right)\right) \subseteq N_{2}(K)$, where $L^{\prime}$ and $L^{\prime \prime}$ are as in Theorem 2.2(1), so that condition $(1)(\mathrm{d})$ is not satisfied and $t$ is a spinor exception. When $s=3$, Theorem 2.1 shows that $\theta\left(O^{+}\left(\langle 8 a, b, c\rangle_{2}\right)\right) \nsubseteq N_{2}(\mathbb{Q}(i))$. For $v_{2}(b)=1$ Theorem 2.1 implies that $\theta\left(O^{+}\left(\left\langle 2 b^{\prime}, c^{\prime}\right\rangle_{2}\right)\right) \subseteq K$ if and only if $K=\mathbb{Q}(\sqrt{-2})$ and $b^{\prime} \equiv c^{\prime}(\bmod 8)$. $K=\mathbb{Q}(\sqrt{-2})$ is equivalent to $2 \nmid v_{2}(a)$ (which implies that $s \in\{4,6, \ldots\}$ ), while $b^{\prime} \equiv c^{\prime}(\bmod 8)$ follows from $2 b^{\prime}+c \equiv \mathcal{S F}\left(a^{\prime} b^{\prime} c^{\prime}\right)(\bmod 8)$, as each of $a^{\prime}, b^{\prime}, c^{\prime}$ is congruent to 1 or $3 \bmod 8$. For $s>4$ we are led to 2-dimensional lattices and Theorem 2.1 implies that $\theta\left(O^{+}\left(\langle 8 a, b, c\rangle_{2}\right)\right) \subseteq N_{2}(\mathbb{Q}(\sqrt{-2}))$ while one sees that none of the conditions of Theorem 2.2(2) can be satisfied, so $t$ is a spinor exception. For $s=4$, Theorem 2.2(2)(a) is satisfied, so $t$ cannot be a spinor exception.

When $v_{2}(b)=2$, Theorem 2.1 implies that $K=\mathbb{Q}(i)$ and hence $v_{2}(a)$ is odd, and Earnest and Hsia [7] showed that we may again consider the spinor norm on 2-dimensional sublattices to get $\theta\left(O^{+}\left(\langle 8 a, b, c\rangle_{2}\right)\right) \subseteq N_{2}(K)$. The conditions in Theorem 2.2(1)(c)-(d) are not satisfied and $L^{\prime}=L_{2}$, so $t$ is a spinor exception.

For $v_{2}(b)=3$, if $t$ is a spinor exception for $Q^{\prime}(x, y, z)$, then Theorem 2.1 for the sublattice $\left\langle 1,8 b^{\prime} c^{\prime}\right\rangle_{2}$ implies that $K=\mathbb{Q}(\sqrt{-2})$ and $b^{\prime} \equiv c^{\prime}(\bmod 8)$. Hence $v_{2}(a)$ must be odd. If $v_{2}(a)=1$, then Theorem 2.2 of Earnest and Hsia 7 implies that $\theta\left(O^{+}\left(\langle 8 a, b, c\rangle_{2}\right)\right)=\mathbb{Q}_{2}^{\times}$. For $v_{2}(a)>1$ odd we may again consider only 2dimensional sublattices and Theorem 2.2(2)(c) is satisfied, so $t$ is a spinor exception for $Q^{\prime}(x, y, z)$. Finally, the property that $t$ is a spinor exception for $Q^{\prime}(x, 2 y, z)$ is equivalent to the case where $r=5$, which was covered above.

For $v_{2}(b)=4$, if $t$ is a spinor exception for $Q^{\prime}(x, y, z)$, then Theorem 2.1] implies that $K=\mathbb{Q}(i)$, and hence $v_{2}(a)$ is odd. Since $\mathcal{S F}\left(a^{\prime} b^{\prime} c^{\prime}\right) \equiv(b+c)^{\prime} \equiv c^{\prime}(\bmod 8)$, we have $a^{\prime} \equiv b^{\prime}(\bmod 8)$ by condition (1), and thus $\theta\left(O^{+}\left(\langle 8 a, b, c\rangle_{2}\right)\right) \subseteq N_{2}(K)$. Moreover, none of the conditions of Theorem 2.2(1) is satisfied, so $t$ is a spinor 
exception. Finally, the property that $t$ is a spinor exception for $Q^{\prime}(x, 2 y, z)$ is equivalent to the case where $r=6$, which was covered above.

Proof of Corollary 1.14, (i) By Theorem 1.2, the form $a x^{2}+2 T_{y}+T_{z}$ is asymptotically universal if and only if $-2 R a^{\prime}$, i.e., each prime divisor of $a^{\prime}$ is congruent to 1 or $3 \bmod 8$.

Now assume that $-2 R a^{\prime}$. As we mentioned before, $\mathcal{S F}\left(a^{\prime}\right)=2 y^{2}+z^{2}$ for some $y, z \in \mathbb{Z}$. Clearly $z$ is odd. If $\mathcal{S F}\left(a^{\prime}\right) \equiv 2+1\left(\bmod 2^{3}\right)$, then $y$ must be odd. Thus we cannot have both (1) and (3) in Theorem 1.13 with $b=2$ and $c=1$. Therefore $a x^{2}+2 T_{y}+T_{z}$ is almost universal.

(ii) By Theorem 1.2 the form $a x^{2}+4 T_{y}+T_{z}$ is asymptotically universal if and only if $-1 R a^{\prime}$, i.e., each prime divisor of $a^{\prime}$ is congruent to $1 \bmod 4$.

Now assume that $-1 R a^{\prime}$. Then $\mathcal{S F}\left(a^{\prime}\right)=4 y^{2}+z^{2}$ for some $y, z \in \mathbb{Z}$. If $\mathcal{S F}\left(a^{\prime}\right) \equiv 4+1\left(\bmod 2^{3}\right)$, then $y$ must be odd. So we cannot have both $(1)$ and (3) in Theorem 1.13 with $b=4$ and $c=1$. It follows that $a x^{2}+4 T_{y}+T_{z}$ is almost universal. We are done.

Proof of Corollary 1.15. Set $f_{k}(x, y, z)=2^{k}\left(x^{2}+T_{y}\right)+m T_{z}$ for $k=0,1,2, \ldots$ By Theorem 1.2, the form $f_{k}$ is asymptotically universal if and only if $-2 R \mathrm{~m}^{\prime}$, and $2 \nmid m$ when $k>0$.

Assume that $f_{k}$ is asymptotically universal. Then all prime divisors of $m^{\prime}$ are congruent to 1 or $3 \bmod 8$; thus $m^{\prime} \equiv 1,3(\bmod 8)$. Note that the equation

$$
8 \times 2^{k} x^{2}+2^{k} y^{2}+m z^{2}=2^{v_{2}\left(2^{k}+m\right)} \mathcal{S F}\left(m^{\prime}\right)
$$

has no integral solutions with $y z$ odd, since the right-hand side of the equation is smaller than $2^{k}+m$.

Case 1. $k=0$. When $a=c=1$ and $b=m$, we obviously have $v_{2}(a)=0,2 \nmid a c$ and $a \equiv c(\bmod 8)$. Thus, if $v_{2}(m) \neq 4,6, \ldots$, then $f_{0}$ is almost universal by Theorem 1.13 for the form $x^{2}+m T_{y}+T_{z}$.

Now suppose $v_{2}(m) \in\{4,6, \ldots\}$. Then $\mathcal{S F}(m) \equiv m+1(\bmod 2)$. Also, $4 \mid m+1$, $v:=v_{2}(m+1)=0, m^{\prime} \equiv \mathcal{S F}\left(m^{\prime}\right)(\bmod 8)$ and $(m+1)^{\prime}=m+1 \equiv 1(\bmod 8)$. By Theorem 1.13 for the form $x^{2}+m T_{y}+T_{z}, f_{0}$ is almost universal if $m^{\prime} \equiv 3(\bmod 8)$, and $f_{0}$ is not almost universal if $m^{\prime} \equiv 1(\bmod 8)$ and $v_{2}(m) \neq 4$.

Case 2. $k>0$. In this case, $m$ is odd. If $k \in\{1,2\}$, then $f_{k}$ is almost universal since (4) in Theorem 1.13 does not hold for $a=b=2^{k}$ and $c=m$. For $k \geq 3$, clearly $4 \nmid 2^{k}+1$ and $\mathcal{S F}\left(m^{\prime}\right)=\mathcal{S} \mathcal{F}(m) \equiv m \equiv 2^{k}+m=\left(2^{k}+m\right)^{\prime}(\bmod 8)$. Applying Theorem 1.13, we find that $f_{k}$ is not almost universal if $k>5$, or $k=3$ and $m \equiv 1(\bmod 8)$.

Combining the above, we have completed the proof.

Proof of Corollary 1.16. Define $g_{k}(x, y, z)=2^{k}\left(x^{2}+2 T_{y}\right)+m T_{z}$ for $k \in \mathbb{N}$. By Theorem 1.2, the form $g_{k}$ is asymptotically universal if and only if $-1 R \mathrm{~m}^{\prime}$ (i.e., all prime divisors of $m^{\prime}$ are congruent to $\left.1 \bmod 4\right)$, and $2 \nmid m$ when $k>0$.

Suppose that $-1 R \mathrm{~m}^{\prime}$. Clearly the equation

$$
8 \times 2^{k} x^{2}+2^{k+1} y^{2}+m z^{2}=2^{v_{2}\left(2^{k+1}+m\right)} \mathcal{S F}\left(m^{\prime}\right)
$$

has no integral solutions with $y$ and $z$ odd (since the right-hand side of the equation is smaller than $\left.2^{k+1}+m\right)$. Note also that if $2 \nmid m$ or $2 \nmid v_{2}(m)$, then $\mathcal{S F}\left(2^{k} 2^{k+1} m\right)=$ 
$\mathcal{S F}(2 m) \not \equiv 2^{k+1}+m(\bmod 2)$, and hence, $(2)$ in Theorem 1.13 holds for the form $g_{k}$.

Case $1 . k=0$. Since $v_{2}(1)-v_{2}(2) \neq 2,4,6, \ldots$, if $v_{2}(m) \leq v_{2}(2)=1$ (i.e., $4 \nmid m$ ), then $x^{2}+2 T_{y}+m T_{z}$ is almost universal by Theorem 1.13. For $a=1, b=m$ and $c=2$, clearly $v_{2}(a) \neq 1,3,5, \ldots$, and $2 \nmid a$ and $a \neq \equiv c(\bmod 8)$. So, by Theorem 1.13 for the form $x^{2}+m T_{y}+2 T_{z}, f_{0}$ is also almost universal when $v_{2}(m) \in\{2,4,6, \ldots\}$. In the case $v_{2}(m) \in\{3,5, \ldots\}$, clearly $\mathcal{S} \mathcal{F}\left(m^{\prime}\right) \equiv m^{\prime} \equiv 1 \equiv m / 2+1=(m+2)^{\prime}(\bmod 4)$, and hence we have (1)-(4) in Theorem 1.13 for the form $x^{2}+m T_{y}+2 T_{z}$. So Theorem 1.13 implies that $g_{0}$ is not almost universal if $v_{2}(m) \in\{5,7, \ldots\}$.

Case 2. $k=1$. As $v_{2}(m+4)=0$ and $\mathcal{S} \mathcal{F}\left(2^{\prime} 4^{\prime} m^{\prime}\right)=\mathcal{S} \mathcal{F}(m) \equiv m \not \equiv(m+$ $4)^{\prime}\left(\bmod 2^{3}\right), g_{1}(x, y, z)=2 x^{2}+4 T_{y}+m T_{z}$ is almost universal by Theorem 1.13

Case 3. $k \geq 2$. In this case, we have (1)-(3) in Theorem 1.13 with $a=2^{k}, b=2^{k+1}$ and $c=m$. Note also that $4 \mid 2^{k}$. So, by Theorem 1.13, $g_{k}$ is not almost universal if $k>2$.

In view of the above, we have proved both (i) and (ii) in Corollary 1.16.

Now we turn to the proof of Theorem 1.17.

Proof of Theorem 1.17. We again start by considering anisotropic primes, again arriving at the fact that only $p=2$ is possible. However, Lemma 3.2 implies bounded divisibility at $p=2$ by the congruence conditions, so there are no relevant anisotropic primes.

We now determine when $t=\mathcal{S} \mathcal{F}\left(a^{\prime} b^{\prime} c^{\prime}\right)$ or $t=2 \mathcal{S F}\left(a^{\prime} b^{\prime} c^{\prime}\right)$ is a spinor exception. For $E(f)$ to be infinite, it is sufficient that $t$ is a spinor exception for $Q^{\prime}(x, y, z)$, while it is necessary that $t$ is a spinor exception for one of the quadratic forms in the inclusion/exclusion.

We will break into cases depending on $v:=v_{2}(a+b+c)$. For $v=2$ and $v_{2}(a)<3$ we have $\theta_{Q}=\theta_{Q^{\prime}}$ and $v_{2}(4 t)=2 \geq s=v_{2}(a)$, so none of the conditions of Theorem 2.2 is satisfied and $t$ is not a spinor exception. When $v=2$ and $v_{2}(a) \geq 3$, we have $4 \mid b+c$. But then, we may assume that $b \equiv 3(\bmod 4)$ without loss of generality, as $b$ and $c$ are both odd. Thus $K=\mathbb{Q}(\sqrt{-2})$ since every prime divisor of $b$ must split in $K$, and hence $v_{2}(t a b c)$ is odd. However, Theorem 2.2(2) implies that $v_{2}(t a b c)$ must be even because $r=v_{2}(b)=0$.

We now must have $v \leq 1, t=2^{v} \mathcal{S F}\left(a^{\prime} b^{\prime} c^{\prime}\right) \equiv(a+b+c)(\bmod 8)$, and $K=$ $K_{2^{v} a b c}$. This gives conditions (1), (2), and (3).

For $v_{2}(b) \geq 5$ we have $\mathcal{S F}\left(a^{\prime} b^{\prime} c^{\prime}\right) \equiv(a+b+c)^{\prime} \equiv c^{\prime}(\bmod 8)$, so $a^{\prime} \equiv b^{\prime}(\bmod 8)$. Again we are led to 2-dimensional sublattices, and it follows that $O^{+}\left(\langle a, b, c\rangle_{2}\right) \subseteq$ $N_{2}(K)$, while none of the conditions of Theorem 2.2 is satisfied, so $t$ is a spinor exception.

For $v_{2}(b)<3$ we have $\theta_{Q}=\theta_{Q^{\prime}(x, y, z)}-\theta_{Q^{\prime}(2 x, y, z)}$. For $b$ odd the sublattice $\langle b, c\rangle_{2}$ gives $b \equiv c(\bmod 8)$ and $K=\mathbb{Q}(i)$, so that $v_{2}(a)$ is odd. But $b \equiv c(\bmod 8)$ automatically by condition (11). If $s=v_{2}(a) \leq 3$, then Theorem 2.2 of Earnest and Hsia [7] implies that $\theta\left(O^{+}\left(\langle a, b, c\rangle_{2}\right)\right)=\mathbb{Q}_{2}^{\times}$so that $t$ is not a spinor exception for $Q^{\prime}$. For $s \geq 5$ Earnest and Hsia showed that we may reduce to 2-dimensional sublattices, so that $\theta\left(O^{+}\left(\langle a, b, c\rangle_{2}\right)\right) \subseteq N_{2}(K)$. We then verify with Theorem 2.1 that the Kneser condition is satisfied for $L^{\prime}$ and $L^{\prime \prime}$ as defined in Theorem 2.2(1). In this case condition (1)(d) of Theorem 2.2 is not satisfied, so $t$ is a spinor exception 
for $Q^{\prime}$. For $s=3$ and $x$ even, the situation is similar to the case $s=5$, thus the above argument shows that $t$ is a spinor exception for $Q^{\prime}(2 x, y, z)$.

When $v_{2}(b)=1$, Theorem 2.1 for the sublattice $\left\langle c, 2 b^{\prime}\right\rangle_{2}$ implies that $K=$ $\mathbb{Q}(\sqrt{-2})$ and $b^{\prime} \equiv c^{\prime}(\bmod 8)$ (which is already satisfied by (10). Thus, $s$ is even. If $s \leq 4$, then Theorem 2.1 for the sublattice $\left\langle c, 2^{s} a^{\prime}\right\rangle_{2}$ gives $5 \in \theta\left(O^{+}\left(\langle a, b, c\rangle_{2}\right)\right)$, so $t$ is not a spinor exception. For $s>4$ we again split into 2-dimensional sublattices, and the Kneser condition is satisfied by Theorem 2.1. The Kneser condition for $L^{\prime}$ as defined in Theorem 2.2(2) is satisfied and condition 2(c) is not satisfied, so $t$ is a spinor exception. Again when $s=4$ we have $s^{\prime}=6$ for $Q^{\prime}(2 x, y, z)$.

When $v_{2}(b)=2$, Theorem 2.1 for the sublattice $\left\langle c, 4 b^{\prime}\right\rangle_{2}$ implies that $K=\mathbb{Q}(i)$, and hence $s \geq 2$ is even. For $s \geq 4$ we may reduce to 2 -dimensional sublattices, and the Kneser condition is satisfied by Theorem 2.1. Theorem 2.2(1)(c)-(d) are not satisfied, so $t$ is a spinor exception. For $s=2$ we have $Q\left(2 x_{1}+x_{2}+x_{3}\right) Q\left(x_{1}\right) / 4 \equiv 3$ $(\bmod 4)$, so that we don't have a spinor exception for $Q^{\prime}$ in this case. However, in the case $s=2$, for $Q^{\prime}(2 x, y, z)$, it follows that $y$ is even by congruence considerations and $r^{\prime}=s^{\prime}=4$. As we will show later, $Q^{\prime}(2 x, y, z)$ has the spinor exception $t$ in this case.

When $v_{2}(b)=3$, by Theorem 2.1 for the sublattice $\left\langle c, 8 b^{\prime}\right\rangle_{2}$, we have $K=$ $\mathbb{Q}(\sqrt{-2})$ and hence $2 \mid s$, as well as $b^{\prime} \equiv c^{\prime}(\bmod 8)$. For $s=4$, Theorem 2.2 of Earnest and Hsia [7] implies that $\theta\left(O^{+}\left(L_{2}\right)\right)=\mathbb{Q}_{2}^{\times}$, so $t$ is not a spinor exception. When $s \geq 6$ we may again consider only 2-dimensional sublattices, and the Kneser condition is satisfied by Theorem 2.1. Theorem 2.2(2)(c) is not satisfied, so $t$ is a spinor exception. For $s=4$ and $x$ even we get a form with $s^{\prime}=6$ and argue as above. For $x, y$ even we get $r^{\prime}=v_{2}(b)+2 \geq 5$, so that $t$ is a spinor exception.

Finally we deal with the case $v_{2}(b)=4$. For $x, y$ even, we have $r^{\prime} \geq 5$, so $t$ is a spinor exception for $Q^{\prime}(2 x, 2 y, z)$. Theorem 2.1 for the sublattice $\left\langle c, 16 b^{\prime}\right\rangle_{2}$ implies that $K=\mathbb{Q}(i)$, and hence $2 \mid s$. But condition (1) implies that $a^{\prime} \equiv b^{\prime}(\bmod 8)$, so we find that for $s$ even, $\theta\left(O^{+}\left(\langle a, b, c\rangle_{2}\right)\right) \subseteq N_{2}(K)$. Since $r=4$ and $s \geq 4$ is even, none of the conditions of Theorem 2.2(1) is satisfied, and $t$ is a spinor exception for $Q^{\prime}(x, y, z)$.

Proof of Corollary 1.18, By Theorem 1.3, the form $a x^{2}+2 T_{y}+T_{z}$ is asymptotically universal if and only if $-2 R a^{\prime}$, i.e., each odd prime divisor of $a$ is congruent to 1 or 3 modulo 8 .

Below we assume that $-2 R a^{\prime}$. As $\min \left\{v_{2}(a), v_{2}(2)\right\} \leq 1,(4)$ in Theorem 1.17 for the form $a x^{2}+2 y^{2}+T_{z}$ just says that $v_{2}(a)-v_{2}(2) \in\{3,5, \ldots\}$.

Now suppose that $v_{2}(a) \in\{4,6, \ldots\}$. Then $v:=v_{2}(a+2+1)=0$ and

$$
\mathcal{S F}\left(a^{\prime} 2^{\prime} 1^{\prime}\right) \equiv(a+3)^{\prime}(\bmod 8) \Longleftrightarrow a^{\prime} \equiv a+3 \equiv 3(\bmod 8)
$$

Also, $\mathcal{S F}(2 a)=2 \mathcal{S F}\left(a^{\prime}\right) \not \equiv a+2+1(\bmod 2)$, and for any odd integers $x, y, z$ we have $a x^{2}+2 y^{2}+z^{2}>a \geq \mathcal{S F}\left(a^{\prime} 2^{\prime} 1^{\prime}\right)$.

In view of the above, (1)-(4) in Theorem 1.17 for the form $a x^{2}+2 y^{2}+T_{z}$ are all valid if and only if $v_{2}(a) \in\{4,6, \ldots\}$ and $a^{\prime} \equiv 3(\bmod 8)$. Thus, by Theorem 1.17 if $a x^{2}+2 y^{2}+T_{z}$ is not almost universal, then we must have $a^{\prime} \equiv 3(\bmod 8)$ and $v_{2}(a) \in\{4,6, \ldots\}$; when $v_{2}(a) \neq 4$ (i.e., $v_{2}(a)-v_{2}(2) \neq 3$ ) the converse also holds.

Combining the above we finally obtain the desired result. 


\section{REFERENCES}

[1] B. C. Berndt, Number Theory in the Spirit of Ramanujan, Amer. Math. Soc., Providence, R.I., 2006. MR 2246314 (2007f:11001)

[2] V. Blomer, G. Harcos, P. Michel, A Burgess-like subconvex bound for twisted $L$-functions (with Appendix 2 by Z. Mao), Forum Math. 19(2007), 61-105. MR2296066 (2008i:11067)

[3] L. E. Dickson, Integers represented by positive ternary quadratic forms, Bull. Amer. Math. Soc. 33(1927), 63-70. MR 1561323

[4] L. E. Dickson, History of the Theory of Numbers, Vol. II, AMS Chelsea Publ., 1999.

[5] W. Duke, Hyperbolic distribution problems and half-integral weight Maass forms, Invent. Math. 92(1988), 73-90. MR931205 (89d:11033)

[6] W. Duke and R. Schulze-Pillot, Representations of integers by positive ternary quadratic forms and equidistribution of lattice points on ellipsoids, Invent. Math. 99(1990), 49-57. MR1029390 (90m:11051)

[7] A. Earnest, J. S. Hsia, Spinor norms of local integral rotations, II, Pacific J. Math. 61(1975), 71-86. MR0404142 (53:7946)

[8] A. Earnest, J. S. Hsia and D. Hung, Primitive representations by spinor genera of ternary quadratic forms, J. London Math. Soc. (2)50(1994), 222-230. MR.1291733 (95k:11044)

[9] S. Guo, H. Pan and Z. W. Sun, Mixed sums of squares and triangular numbers (II), Integers 7(2007), \# A56, 5pp. MR2373118 (2008m:11078)

[10] H. Iwaniec, Fourier coefficients of modular forms of half-integral weight, Invent. Math. 87(1987), 385-401. MR870736 (88b:11024)

[11] B. Jones, The Arithmetic Theory of Quadratic Forms, Math. Assoc. Amer., Carus Math. Mono. 10, Buffalo, New York, 1950. MR0037321(12:244a)

[12] B. Kane, On two conjectures about mixed sums of squares and triangular numbers, J. Combin. Number Theory 1(2009), 77-90.

[13] H. D. Kloosterman, On the representation of numbers in the form $a x^{2}+b y^{2}+c z^{2}+d t^{2}$, Acta Math. 49(1926), 407-464.

[14] M. Kneser, Darstellungsmasse indefiniter quadratischer Formen, Math. Z. 11(1961), 188194. MR0140487 (25:3907)

[15] W. Kohnen, Fourier coefficients of modular forms of half-integral weight, Math. Ann. 271(1985), 237-268. MR783554 (86i:11018)

[16] B. K. Oh and Z. W. Sun, Mixed sums of squares and triangular numbers (III), J. Number Theory 129(2009), 964-969. MR2499416

[17] O. T. O'Meara, Introduction to Quadratic Forms, Springer, New York, 1963.

[18] K. Ono, Web of Modularity: Arithmetic of the Coefficients of Modular Forms and Q-series, Amer. Math. Soc., Providence, R.I., 2003. MR2020489 (2005c:11053)

[19] K. Ono, Honoring a gift from Kumbakonam, Notices Amer. Math. Soc. 53(2006), 640-651. MR2235326

[20] K. Ono and K. Soundararajan, Ramanujan's ternary quadratic form, Invent. Math. 130(1997), 415-454. MR 1483991 (99b:11036)

[21] L. Panaitopol, On the representation of natural numbers as sums of squares, Amer. Math. Monthly 112(2005), 168-171. MR2121327(2005k:11077)

[22] S. Ramanujan, On the expression of a number in the form $a x^{2}+b y^{2}+c z^{2}+d u^{2}$, Proc. Camb. Philo. Soc. 19(1916), 11-21.

[23] R. Schulze-Pillot, Representations by integral quadratic forms - A Survey, in: Algebraic and Arithmetic Theory of Quadratic Forms, pp. 303-321, Contemp. Math., 344, Amer. Math. Soc., Providence, R.I., 2004. MR2060206 (2005g:11057)

[24] R. Schulze-Pillot, Darstellung durch Spinorgeschlechter ternarer quadratischer Formen, J. Number Theory 12(1980), 529-540. MR.599822 (82k:10024)

[25] R. Schulze-Pillot, Exceptional integers for genera of integral ternary positive definite quadratic forms, Duke Math. J. 102(2000), 351-357. MR.1749442 (2001a:11068)

[26] C. L. Siegel, Über die Klassenzahl algebraischer Zahlkörper, Acta Arith. 1(1935), 83-86.

[27] Z. W. Sun, Mixed sums of squares and triangular numbers, Acta. Arith. 127(2007), 103-113. MR2289977 (2007m:11052)

[28] Z. W. Sun, A message to Number Theory Mailing List, April 27, 2008. http://1istserv. nodak. edu/cgi-bin/wa . exe?A2=ind0804\&L=nmbrthry\&T=0\&P=1670 
[29] Z. W. Sun, On sums of primes and triangular numbers, J. Combin. Number Theory 1(2009), $65-76$.

[30] J. L. Waldspurger, Sur les coefficients de Fourier des formes modulaires de poids demi-entier, J. Math. Pures Appl. 60(1981), 375-484. MR646366 (83h:10061)

Department of Mathematics, University of Cologne, Weyertal 86-90, 50931 Cologne, Germany

E-mail address: bkane@math.uni-koeln.de

Department of Mathematics, Nanjing University, Nanjing 210093, People's Republic OF CHINA

E-mail address: zwsun@nju.edu.cn 